\title{
Interacting reinforced stochastic processes: Statistical inference based on the weighted empirical means
}

\author{
GIACOMO ALETTI ${ }^{1}$, IRENE CRIMALDI ${ }^{2}$ and ANDREA GHIGLIETTI ${ }^{3}$ \\ ${ }^{1}$ ADAMSS Center, Università degli Studi di Milano, Milan, Italy.E-mail: giacomo.aletti@unimi.it \\ ${ }^{2}$ IMT School for Advanced Studies, Lucca, Italy.E-mail: irene.crimaldi@imtlucca.it \\ ${ }^{3}$ LivaNova, Milan, Italy.E-mail: Andrea.Ghiglietti@guest.unimi.it
}

This work deals with a system of interacting reinforced stochastic processes, where each process $X^{j}=$ $\left(X_{n, j}\right)_{n}$ is located at a vertex $j$ of a finite weighted directed graph, and it can be interpreted as the sequence of "actions" adopted by an agent $j$ of the network. The interaction among the dynamics of these processes depends on the weighted adjacency matrix $W$ associated to the underlying graph: indeed, the probability that an agent $j$ chooses a certain action depends on its personal "inclination" $Z_{n, j}$ and on the inclinations $Z_{n, h}$, with $h \neq j$, of the other agents according to the entries of $W$. The best known example of reinforced stochastic process is the Pólya urn.

The present paper focuses on the weighted empirical means $N_{n, j}=\sum_{k=1}^{n} q_{n, k} X_{k, j}$, since, for example, the current experience is more important than the past one in reinforced learning. Their almost sure synchronization and some central limit theorems in the sense of stable convergence are proven. The new approach with weighted means highlights the key points in proving some recent results for the personal inclinations $Z^{j}=\left(Z_{n, j}\right)_{n}$ and for the empirical means $\bar{X}^{j}=\left(\sum_{k=1}^{n} X_{k, j} / n\right)_{n}$ given in recent papers (e.g. Aletti, Crimaldi and Ghiglietti (2019), Ann. Appl. Probab. 27 (2017) 3787-3844, Crimaldi et al. Stochastic Process. Appl. 129 (2019) 70-101). In fact, with a more sophisticated decomposition of the considered processes, we can understand how the different convergence rates of the involved stochastic processes combine. From an application point of view, we provide confidence intervals for the common limit inclination of the agents and a test statistics to make inference on the matrix $W$, based on the weighted empirical means. In particular, we answer a research question posed in Aletti, Crimaldi and Ghiglietti (2019).

Keywords: asymptotic normality; complex networks; interacting random systems; reinforced learning; reinforced stochastic processes; synchronization; urn models; weighted empirical means

\section{Framework, model and motivations}

The stochastic evolution of systems composed by elements which interact among each other has always been of great interest in several scientific fields. For example, economic and social sciences deal with agents that take decisions under the influence of other agents. In social life, preferences and beliefs are partly transmitted by means of various forms of social interaction and opinions are driven by the tendency of individuals to become more similar when they interact. Hence, a collective phenomenon, that we call "synchronization", reflects the result of the interactions among different individuals. The underlying idea is that individuals have opinions that change according to the influence of other individuals giving rise to a sort of collective behavior. 
In particular, there exists a growing interest in systems of interacting urn models (see, e.g., Aletti and Ghiglietti [4], Benaïm et al. [7], Chen and Lucas [10], Cirillo, Gallegati and Hüsler [12], Crimaldi, Dai Pra and Minelli [18], Crimaldi et al. [21], Fortini, Petrone and Sporysheva [23], Hayhoe, Alajaji and Gharesifard [27], Lima [29], Paganoni and Secchi [32]) and their variants and generalizations (see, e.g., Aletti, Crimaldi and Ghiglietti [1,2], Crimaldi et al. [17]). Our work is placed in the stream of this scientific literature. Specifically, it deals with the class of the so-called interacting reinforced stochastic processes considered in Aletti, Crimaldi and Ghiglietti [2], Aletti, Crimaldi and Ghiglietti [1] with a general network-based interaction and in Crimaldi et al. [17] with a mean-field interaction. Generally speaking, by reinforcement in a stochastic dynamics we mean any mechanism for which the probability that a given event occurs has an increasing dependence on the number of times that the same event occurred in the past. This "reinforcement mechanism", also known as "preferential attachment rule" or "Rich get richer rule" or "Matthew effect", is a key feature governing the dynamics of many biological, economic and social systems (see, e.g., Pemantle [33]). The best known example of reinforced stochastic process is the standard Eggenberger-Pólya urn (see Eggenberger and Pólya [22], Mahmoud [30]), which has been widely studied and generalized (some recent variants can be found in Aletti, Ghiglietti and Rosenberger [5], Aletti, Ghiglietti and Vidyashankar [6], Berti et al. [9], Chen and Kuba [11], Collevecchio, Cotar and LiCalzi [13], Crimaldi [15], Ghiglietti and Paganoni [24], Ghiglietti, Vidyashankar and Rosenberger [25], Laruelle and Pagès [28]).

A Reinforced Stochastic Process (RSP) can be defined as a stochastic process in which, along the time-steps, an agent performs an action chosen in the set $\{0,1\}$ in such a way that the probability of adopting "action 1" at a certain time-step has an increasing dependence on the number of times that the agent adopted "action 1" in the previous actions. Formally, we define it as a stochastic process $X=\left\{X_{n}: n \geq 1\right\}$ taking values in $\{0,1\}$ and such that

$$
P\left(X_{n+1}=1 \mid Z_{0}, X_{1}, \ldots, X_{n}\right)=Z_{n},
$$

with

$$
Z_{n}=\left(1-r_{n-1}\right) Z_{n-1}+r_{n-1} X_{n},
$$

where $Z_{0}$ is a random variable with values in $[0,1]$ and $\left(r_{n}\right)_{n \geq 0}$ is a sequence of real numbers in $(0,1)$. We will focus on the case when

$$
\lim _{n} n^{\gamma} r_{n}=c>0 \quad \text { with } 1 / 2<\gamma \leq 1
$$

(We refer to Crimaldi et al. [17] for a discussion on the case $0<\gamma \leq 1 / 2$, for which there is a different asymptotic behavior of the model that is out of the scope of this research work.) The process $X$ describes the sequence of actions along the time-steps and, if at time-step $n$, the "action 1" has taken place, that is $X_{n}=1$, then for "action 1" the probability of occurrence at time-step $(n+1)$ increases. Therefore, the larger $Z_{n-1}$, the higher the probability of having $X_{n}=1$ and so the higher the probability of having $Z_{n}$ greater than $Z_{n-1}$. As a consequence, the larger the number of times in which $X_{k}=1$ with $1 \leq k \leq n$, the higher the probability $Z_{n}$ of observing $X_{n+1}=1$. 
As told before, the best known example of reinforced stochastic process is the standard Eggenberger-Pólya urn, where an urn contains $a$ red and $b$ white balls and, at each discrete time, a ball is drawn out from the urn and then it is put again inside the urn together with one additional ball of the same color. In this case, we have $Z_{0}=\frac{a}{a+b}$ and $Z_{n}=\frac{a+\sum_{k=1}^{n} X_{k}}{a+b+n}$ for $n \geq 1$ and it is immediate to verify that $Z_{n+1}=\left(1-r_{n}\right) Z_{n}+r_{n} X_{n+1}$ with $r_{n}=(a+b+n+1)^{-1}$ and so $\gamma=c=1$.

In the present work, we are interested in the analysis of a system of $N \geq 2$ interacting reinforced stochastic processes $\left\{X^{j}=\left(X_{n, j}\right)_{n \geq 1}: 1 \leq j \leq N\right\}$ positioned at the vertices of a weighted directed graph $G=(V, E, W)$, where $V:=\{1, \ldots, N\}$ denotes the set of vertices, $E \subseteq V \times V$ the set of edges and $W=\left[w_{h, j}\right]_{h, j \in V \times V}$ the weighted adjacency matrix with $w_{h, j} \geq 0$ for each pair of vertices. The presence of the edge $(h, j) \in E$ indicates a "direct influence" that the vertex $h$ has on the vertex $j$ and it corresponds to a strictly positive element $w_{h, j}$ of $W$, that represents a weight quantifying this influence. We assume the weights to be normalized so that $\sum_{h=1}^{N} w_{h, j}=1$ for each $j \in V$. The interaction between the processes $\left\{X^{j}: j \in V\right\}$ is explicitly inserted in Equation (1.1) and it is modeled as follows: for any $n \geq 0$, the random variables $\left\{X_{n+1, j}: j \in V\right\}$ are conditionally independent given $\mathcal{F}_{n}$ with

$$
P\left(X_{n+1, j}=1 \mid \mathcal{F}_{n}\right)=\sum_{h=1}^{N} w_{h, j} Z_{n, h}=w_{j j} Z_{n, j}+\sum_{h \neq j} w_{h, j} Z_{n, h},
$$

where $\mathcal{F}_{n}:=\sigma\left(Z_{0, h}: h \in V\right) \vee \sigma\left(X_{k, j}: 1 \leq k \leq n, j \in V\right)$ and, for each $h \in V$, the evolution dynamics of the single process $\left(Z_{n, h}\right)_{n \geq 0}$ is the same as in (1.2), that is

$$
Z_{n, h}=\left(1-r_{n-1}\right) Z_{n-1, h}+r_{n-1} X_{n, h},
$$

with $Z_{0, h}$ a random variable taking values in $[0,1]$ and $\left(r_{n}\right)_{n \geq 0}$ a sequence of real numbers in $(0,1)$ such that condition (1.3) holds true.

As an example, we can imagine that $G=(V, E)$ represents a network of $N$ individuals that at each time-step have to make a choice between two possible actions $\{0,1\}$. For any $n \geq 1$, the random variables $\left\{X_{n, j}: j \in V\right\}$ take values in $\{0,1\}$ and they describe the actions adopted by the agents of the network along the time-steps; while each random variable $Z_{n, h}$ takes values in $[0,1]$ and it can be interpreted as the "personal inclination" of the agent $h$ of adopting "action 1". Thus, the probability that the agent $j$ adopts "action 1" at time-step $(n+1)$ is given by a convex combination of $j$ 's own inclination and the inclination of the other agents at time-step $n$, according to the "influence-weights" $w_{h, j}$ as in (1.4). Note that, from a mathematical point of view, we can have $w_{j j} \neq 0$ or $w_{j j}=0$. In both cases, we have a reinforcement mechanism for the personal inclinations of the agents: indeed, by (1.5), whenever $X_{n, h}=1$, we have a positive increment in the personal inclination of the agent $h$, that is $Z_{n, h} \geq Z_{n-1, h}$. However, only in the case $w_{j j}>0$, this fact results in a greater probability of having $X_{n+1, j}=1$ according to (1.4). Therefore, if $w_{j j}>0$, then we have a "true self-reinforcing" mechanism; while, in the opposite case, we have a reinforcement property only in the own inclination of the single agent, but this does not affect the probability (1.4).

The literature Aletti, Crimaldi and Ghiglietti [1], Crimaldi et al. [17], Crimaldi, Dai Pra and Minelli [18], Crimaldi et al. [21] focus on the asymptotic behavior of the stochastic processes 
of the personal inclinations $\left\{Z^{j}=\left(Z_{n, j}\right)_{n}: j \in V\right\}$ of the agents; while Aletti, Crimaldi and Ghiglietti [2] studies the average of times in which the agents adopt "action 1", that is, the stochastic processes of the empirical means $\left\{\bar{X}_{n}^{j}=\left(\frac{1}{n} \sum_{k=1}^{n} X_{k, j}\right)_{n}: j \in V\right\}$. The results given in Aletti, Crimaldi and Ghiglietti [2], together with the resulting statistical tools, represent a great improvement in any area of application, since the personal inclinations $Z_{n, j}$ of the agents are usually latent variables, while the empirical means $\bar{X}_{n}^{j}$ of the actions adopted by the agents are likely to be observed.

In the present paper, we continue in this direction: indeed, motivated, for instance, by the fact that the current experience is more important than the past one in reinforced learning, we here study the asymptotic behaviors of the "weighted" empirical means. Moreover, using a more sophisticated decomposition of the involved processes, we can handle here also the case $\gamma<1$, which was in part left open, and we solve a research question posed in Aletti, Crimaldi and Ghiglietti [2]. Consequently, we succeed in constructing a test statistics to make inference on the weighted adjacency matrix $W$ of the network for all values of the model parameters (not only in the case $\gamma=1$, see Remark 4.2 for the details). More precisely, in this paper we focus on the weighted average of times in which the agents adopt "action 1", that is, we study the stochastic processes of the weighted empirical means $\left\{N^{j}=\left(N_{n, j}\right)_{n}: j \in V\right\}$ defined, for each $j \in V$, as $N_{0}^{j}:=0$ and, for any $n \geq 1$,

$$
N_{n, j}:=\sum_{k=1}^{n} q_{n, k} X_{k, j}, \quad \text { where } q_{n, k}:=\frac{a_{k}}{\sum_{l=1}^{n} a_{l}},
$$

with $\left(a_{k}\right)_{k \geq 1}$ a suitable sequence of strictly positive real numbers. (In particular, if, according to the principle of reinforced learning, we want to give more "weight" to the current, or more recent, experience, we can choose $\left(a_{k}\right)_{k \geq 1}$ increasing.) Since $\sum_{k=1}^{n} q_{n, k}=1$, we have the relation

$$
\sum_{k=1}^{n-1} q_{n, k} X_{k, j}=\frac{\sum_{l=1}^{n-1} a_{l}}{\sum_{l=1}^{n} a_{l}}\left(\sum_{k=1}^{n-1} q_{n-1, k} X_{k, j}\right)=\left(1-q_{n, n}\right) N_{n-1, j}
$$

and so we get

$$
N_{n, j}=\left(1-q_{n, n}\right) N_{n-1, j}+q_{n, n} X_{n, j} .
$$

The above dynamics (1.4), (1.5) and (1.7) can be expressed in a compact form, using the random vectors $\mathbf{X}_{n}:=\left(X_{n, 1}, \ldots, X_{n, N}\right)^{\top}$ for $n \geq 1, \mathbf{N}_{n}:=\left(N_{n, 1}, \ldots, N_{n, N}\right)^{\top}$ and $\mathbf{Z}_{n}:=$ $\left(Z_{n, 1}, \ldots, Z_{n, N}\right)^{\top}$ for $n \geq 0$, as

$$
E\left[\mathbf{X}_{n+1} \mid \mathcal{F}_{n}\right]=W^{\top} \mathbf{Z}_{n},
$$

where $W^{\top} \mathbf{1}=\mathbf{1}$ by the normalization of the weights, and

$$
\left\{\begin{array}{l}
\mathbf{Z}_{n}=\left(1-r_{n-1}\right) \mathbf{Z}_{n-1}+r_{n-1} \mathbf{X}_{n}, \\
\mathbf{N}_{n}=\left(1-q_{n, n}\right) \mathbf{N}_{n-1}+q_{n, n} \mathbf{X}_{n}
\end{array}\right.
$$


Under suitable assumptions, we prove the almost sure synchronization of the stochastic processes $N^{j}=\left(N_{n, j}\right)_{n}$, with $j \in V$, toward the same limit random variable $Z_{\infty}$, which is also the common limit random variable of the stochastic processes $Z^{j}=\left(Z_{n, j}\right)_{n}$, and we provide some CLTs in the sense of stable convergence. In particular, we assume

$$
\lim _{n} n^{v} q_{n, n}=q>0 \quad \text { with } 1 / 2<v \leq 1
$$

and the asymptotic covariances in the provided CLTs depend on the random variable $Z_{\infty}$, on the eigen-structure of the weighted adjacency matrix $W$ and on the parameters $\gamma, c$ and $v, q$ governing the asymptotic behavior of the sequence $\left(r_{n}\right)_{n}$ and $\left(q_{n, n}\right)_{n}$, respectively. We also discuss the possible statistical applications of these convergence results: asymptotic confidence intervals for the common limit random variable $Z_{\infty}$ and test statistics to make inference on the weighted adjacency matrix $W$ of the network. In particular, as said before, we obtain a statistical test on the matrix $W$ for all values of the model parameters. Moreover, our results give a hint regarding a possible "optimal choice" of $v$ and $q$ and so point out the advantages of employing the weighted empirical means with $v<1$, instead of the simple empirical means.

Finally, we point out that the existence of joint central limit theorems for the pair $\left(\mathbf{Z}_{n}, \mathbf{N}_{n}\right)$ is not obvious because the "discount factors" in the dynamics of the increments $\left(\mathbf{Z}_{n}-\mathbf{Z}_{n-1}\right)_{n}$ and $\left(\mathbf{N}_{n}-\mathbf{N}_{n-1}\right)_{n}$ are generally different. Indeed, as shown in (1.9), these two stochastic processes follow the dynamics

$$
\left\{\begin{array}{l}
\mathbf{Z}_{n}-\mathbf{Z}_{n-1}=r_{n-1}\left(\mathbf{X}_{n}-\mathbf{Z}_{n-1}\right), \\
\mathbf{N}_{n}-\mathbf{N}_{n-1}=q_{n, n}\left(\mathbf{X}_{n}-\mathbf{N}_{n-1}\right),
\end{array}\right.
$$

and so, when we assume $v \neq \gamma$, it could be surprising that in some cases there exists a common convergence rate for the pair $\left(\mathbf{Z}_{n}, \mathbf{N}_{n}\right)$. It is worthwhile to note that dynamics similar to (1.11) have already been considered in the Stochastic Approximation literature. Specifically, in Mokkadem and Pelletier [31] the authors established a CLT for a pair of recursive procedures having two different step-sizes. However, this result does not apply to our situation. Indeed, the covariance matrices $\Sigma_{\mu}$ and $\Sigma_{\theta}$ in their main result (Theorem 1) are deterministic, while the asymptotic covariance matrices in our CLTs are random (as said before, they depend on the random variable $Z_{\infty}$ ). This is why we do not use the simple convergence in distribution, but we employ the notion of stable convergence, which is, among other things, essential for the considered statistical applications. Moreover in Mokkadem and Pelletier [31], the authors find two different convergence rates, depending on the two different step-sizes, while, as already said, we find a common convergence rate also in some cases with $v \neq \gamma$.

Summing up, this work shows convergence results for the stochastic processes of the personal inclinations $Z^{j}=\left(Z_{n, j}\right)_{n}$ and of the weighted empirical means $N^{j}=\left(N_{n, j}\right)_{n}$. As a byproduct, we are able to complete some convergence results obtained in Aletti, Crimaldi and Ghiglietti [2], Aletti, Crimaldi and Ghiglietti [1], where the sole personal inclinations or the empirical means $\bar{X}^{j}=\left(\bar{X}_{n, j}\right)_{n}$ were considered. The main focus here concerns the new decomposition employed for the analysis of the asymptotic behavior of the pair $\left(\mathbf{Z}_{n}, \mathbf{N}_{n}\right)$, that, among other things, allows us to solve the research question arisen in Aletti, Crimaldi and Ghiglietti [2] regarding the statistical test on $W$ in the case $\gamma<1$. Thus, in what follows, we will go fast on the points in common with Aletti, Crimaldi and Ghiglietti [1,2], while we will concentrate on the novelties. 
The rest of the paper is organized as follows. In Section 2, we describe the notation and the assumptions used along the paper. In Section 3 and Section 4, we illustrate our main results and we discuss some possible statistical applications. An interesting example of interacting system is also provided in order to clarify the statement of the theorems and the related comments. Section 5 and Section 6 contain the proofs or the main steps of the proofs of our results, while the technical details have been gathered in the supplementary material (Aletti, Crimaldi and Ghiglietti [3]). In particular, Section 5.2 contains the main ingredient of the proofs of the CLTs, that is a suitable decomposition of the joint stochastic process $\left(\mathbf{Z}_{n}, \mathbf{N}_{n}\right)$. Finally, for the reader's convenience, the appendix supplies a brief review on the notion of stable convergence and its variants (see, e.g., Crimaldi [14,16], Crimaldi, Letta and Pratelli [19], Hall and Heyde [26], Zhang [34]) and the statements of some technical lemmas quoted in the paper.

\section{Notation and assumptions}

Throughout all the paper, we will assume $N \geq 2$ and adopt the same notation used in Aletti, Crimaldi and Ghiglietti [1,2]. In particular, we denote by $\mathcal{R e}(z), \mathcal{I m}(z), \bar{z}$ and $|z|$ the real part, the imaginary part, the conjugate and the modulus of a complex number $z$. Then, for a matrix $A$ with complex elements, we let $\bar{A}$ and $A^{\top}$ be its conjugate and its transpose, while we indicate by $|A|$ the sum of the modulus of its elements. The identity matrix is denoted by $I$, independently of its dimension that will be clear from the context. The spectrum of $A$, that is, the set of all the eigenvalues of $A$ repeated with their multiplicity, is denoted by $\operatorname{Sp}(A)$, while its sub-set containing the eigenvalues with maximum real part is denoted by $\lambda_{\max }(A)$, i.e. $\lambda^{*} \in \lambda_{\max }(A)$ whenever $\mathcal{R e}\left(\lambda^{*}\right)=\max \{\mathcal{R e}(\lambda): \lambda \in \operatorname{Sp}(A)\}$. The notation $\operatorname{diag}\left(a_{1}, \ldots, a_{d}\right)$ indicates the diagonal matrix of dimension $d$ with diagonal elements $a_{1}, \ldots, a_{d}$. Finally, we consider any vector $\mathbf{v}$ as a matrix with only one column (so that all the above notations apply to $\mathbf{v}$ ) and we indicate by $\|\mathbf{v}\|$ its norm, that is, $\|\mathbf{v}\|^{2}=\overline{\mathbf{v}}^{\top} \mathbf{v}$. The vectors and the matrices whose elements are all ones or zeros are denoted by $\mathbf{1}$ and $\mathbf{0}$, respectively, independently of their dimension that will be clear from the context.

For the matrix $W$, we make the following assumption:

Assumption 2.1. The weighted adjacency matrix $W$ is irreducible and diagonalizable.

The irreducibility of $W$ reflects a situation in which all the vertices are connected among each others and hence there are no sub-systems with independent dynamics (see Aletti, Crimaldi and Ghiglietti [1], Aletti and Ghiglietti [4] for further details). The diagonalizability of $W$ allows us to find a non-singular matrix $\widetilde{U}$ such that $\widetilde{U}^{\top} W\left(\widetilde{U}^{\top}\right)^{-1}$ is diagonal with complex elements $\lambda_{j} \in \operatorname{Sp}(W)$. Notice that each column $\mathbf{u}_{j}$ of $\widetilde{U}$ is a left eigenvector of $W$ associated to the eigenvalue $\lambda_{j}$. Without loss of generality, we take $\left\|\mathbf{u}_{j}\right\|=1$. Moreover, when the multiplicity of some $\lambda_{j}$ is bigger than one, we set the corresponding eigenvectors to be orthogonal. Then, if we define $\widetilde{V}=\left(\widetilde{U}^{\top}\right)^{-1}$, we have that each column $\mathbf{v}_{j}$ of $\widetilde{V}$ is a right eigenvector of $W$ associated to the 
eigenvalue $\lambda_{j}$ such that

$$
\mathbf{u}_{j}^{\top} \mathbf{v}_{j}=1, \quad \text { and } \quad \mathbf{u}_{h}^{\top} \mathbf{v}_{j}=0, \quad \forall h \neq j
$$

These constraints combined with the above assumptions on $W$ (precisely, $w_{h, j} \geq 0, W^{\top} \mathbf{1}=\mathbf{1}$ and the irreducibility) imply, by Frobenius-Perron theorem, that $\lambda_{1}:=1$ is an eigenvalue of $W$ with multiplicity one, $\lambda_{\max }(W)=\{1\}$ and

$$
\mathbf{u}_{1}=N^{-1 / 2} \mathbf{1}, \quad N^{-1 / 2} \mathbf{1}^{\top} \mathbf{v}_{1}=1 \quad \text { and } \quad \forall 1 \leq j \leq N \quad v_{1, j}:=\left[\mathbf{v}_{1}\right]_{j}>0 .
$$

Moreover, we recall the relation

$$
\sum_{j=1}^{N} \mathbf{u}_{j} \mathbf{v}_{j}^{\top}=I
$$

Finally, we set $\alpha_{j}:=1-\lambda_{j} \in \mathbb{C}$ for each $j \geq 2$, i.e. for each $\lambda_{j}$ belonging to $\operatorname{Sp}(W) \backslash\{1\}$, and we denote by $\lambda^{*}$ an eigenvalue belonging to $\operatorname{Sp}(W) \backslash\{1\} \operatorname{such}$ that $\mathcal{R e}\left(\lambda^{*}\right)=\max \left\{\mathcal{R e}\left(\lambda_{j}\right): \lambda_{j} \in\right.$ $\operatorname{Sp}(W) \backslash\{1\}\}$.

Throughout all the paper, we assume that the two sequences $\left(r_{n}\right)_{n \geq 0}$ and $\left(q_{n, n}\right)_{n \geq 1}$, which appear in (1.9), satisfy the following assumption:

Assumption 2.2. There exist real constants $\gamma, v \in(1 / 2,1]$ and $c, q>0$ such that

$$
r_{n-1}=\frac{c}{n^{\gamma}}+O\left(\frac{1}{n^{2 \gamma}}\right) \text { and } q_{n, n}=\frac{q}{n^{v}}+O\left(\frac{1}{n^{2 v}}\right) .
$$

In particular, it follows $\lim _{n} n^{\gamma} r_{n}=c>0$ and $\lim _{n} n^{v} q_{n, n}=q>0$. The following remark will be useful for a certain proof in the sequel.

Remark 2.1. Recalling that $q_{n, n}=a_{n} / \sum_{l=1}^{n} a_{l}$, the second relation in (2.4) implies that $\sum_{n=1}^{+\infty} a_{n}=+\infty$. Indeed, that relation together with $\sum_{n=1}^{+\infty} a_{n}=\ell<+\infty$ entails $a_{n}=q \ell n^{-v}+$ $O\left(n^{-2 v}\right)$ and so, since $v \leq 1, \sum_{n=1}^{+\infty} a_{n}=+\infty$, which is a contradiction.

In the special case of $a_{n}=1$ for each $n$, the random variables $N_{n, j}$ correspond to the simple empirical means and we have $v=1$ and $q=1$. Other possible choices are the following:

- $\sum_{l=1}^{n} a_{l}=n^{\delta}$ with $\delta>0$, which brings to $a_{n}=n^{\delta}-(n-1)^{\delta}$ and

$$
q_{n, n}=1-\frac{\sum_{l=1}^{n-1} a_{l}}{\sum_{l=1}^{n} a_{l}}=1-\left(1-\frac{1}{n}\right)^{\delta}=\delta n^{-1}+O\left(n^{-2}\right),
$$

so that we have $v=1$ and $q=\delta>0$; 
- $\sum_{l=1}^{n} a_{l}=\exp \left(b n^{\delta}\right)$ with $b>0$ and $\delta \in(0,1 / 2)$, which brings to $a_{n}=\exp \left(b n^{\delta}\right)-$ $\exp \left(b(n-1)^{\delta}\right)$ and

$$
\begin{aligned}
q_{n, n} & =1-\frac{\sum_{l=1}^{n-1} a_{l}}{\sum_{l=1}^{n} a_{l}} \\
& =1-\exp \left[b\left((n-1)^{\delta}-n^{\delta}\right)\right] \\
& =b n^{\delta}\left(1-\left(1-n^{-1}\right)^{\delta}\right)+O\left(n^{2 \delta}\left(1-\left(1-n^{-1}\right)^{\delta}\right)^{2}\right) \\
& =b n^{\delta}\left(\delta n^{-1}+O\left(n^{-2}\right)\right)+O\left(n^{-(2-2 \delta)}\right) \\
& =b \delta n^{-(1-\delta)}+O\left(n^{-(2-\delta)}\right)+O\left(n^{-(2-2 \delta)}\right) \\
& =b \delta n^{-(1-\delta)}+O\left(n^{-2(1-\delta)}\right)
\end{aligned}
$$

so that $v=(1-\delta) \in(1 / 2,1)$ and $q=b \delta>0$.

To ease the notation, we set $\widehat{r}_{n-1}:=c n^{-\gamma}$ and $\widehat{q}_{n, n}:=q n^{-v}$, so that condition (2.4) can be rewritten as

$$
r_{n-1}=\widehat{r}_{n-1}+O\left(\frac{1}{n^{2 \gamma}}\right) \text { and } q_{n, n}=\widehat{q}_{n, n}+O\left(\frac{1}{n^{2 v}}\right) .
$$

For the CLTs provided in the sequel, we make also the following assumption:

Assumption 2.3. When $\gamma=1$, we assume the condition $c>1 /\left[2\left(1-\mathcal{R e}\left(\lambda^{*}\right)\right)\right]$, i.e. $\operatorname{Re}\left(\lambda^{*}\right)<$ $1-(2 c)^{-1}$. When $v=1$, we assume $q>1 / 2$.

Note that in Assumption 2.2 condition (2.4) for the sequence $\left(r_{n}\right)_{n}$ is slightly more restrictive than the one assumed in Aletti, Crimaldi and Ghiglietti [1,2]. However, it is always verified in the applicative contexts we have in mind. The reason behind this choice is that we want to avoid some technical complications in order to focus on the differences brought by the use of the weighted empirical means, specially on the relationship between the pair $(\gamma, v)$ and the asymptotic behaviors of the considered stochastic processes. For the same reason, in the CLTs for the case $v=\gamma$, we add also the following assumption:

$$
\forall j \geq 2 \quad q \neq c \alpha_{j}
$$

We think that this condition is not necessary. Indeed, if there exists $j \geq 2$ such that $q=c \alpha_{j}$, we conjecture that our proofs still work (but using in the proof of Lemma 5.1 the appropriate asymptotic expression of a certain quantity, called $G_{k+1, n-1}(x, q)$, whose asymptotic behavior is completely described in the supplementary material (Aletti, Crimaldi and Ghiglietti [3], Section A.3)) and they lead to exactly the same asymptotic covariances provided in the CLTs under the above condition (2.5). Our conjecture is motivated by the fact that this is what happens in Aletti, Crimaldi and Ghiglietti [2] for the simple empirical means. Moreover, the expressions obtained for the asymptotic covariances in the following CLTs do not require condition (2.5). However, as told before, we do not want to make the following proofs even heavier and so, when $v=\gamma$, we will work under condition (2.5). 


\section{Main results on the joint stochastic process}

The first achievement concerns the almost sure synchronization of all the involved stochastic processes, that is

$$
\mathbf{Y}_{n}:=\left(\begin{array}{l}
\mathbf{Z}_{n} \\
\mathbf{N}_{n}
\end{array}\right) \stackrel{\text { a.s. }}{\longrightarrow} Z_{\infty} \mathbf{1},
$$

where $Z_{\infty}$ is a random variable with values in $[0,1]$. This fact means that all the stochastic processes $Z^{j}=\left(Z_{n, j}\right)_{n}$ and $N^{j}=\left(N_{n, j}\right)_{n}$ positioned at different vertices $j \in V$ of the graph converge almost surely to the same random variable $Z_{\infty}$.

The synchronization for the first component of $\mathbf{Y}_{n}$, that is

$$
\left[\mathbf{Y}_{n}\right]_{1}=\mathbf{Z}_{n} \stackrel{\text { a.s. }}{\longrightarrow} Z_{\infty} \mathbf{1},
$$

is the result contained in Aletti, Crimaldi and Ghiglietti [1], Theorem 3.1, while for the second component, we prove in the present work the following result.

Theorem 3.1. Under Assumptions 2.1 and 2.2, we have

$$
\left[\mathbf{Y}_{n}\right]_{2}=\mathbf{N}_{n} \stackrel{\text { a.s. }}{\longrightarrow} Z_{\infty} \mathbf{1} .
$$

Regarding the distribution of $Z_{\infty}$, we recall that Aletti, Crimaldi and Ghiglietti ([1], Theorems 3.5 and 3.6) state the following two properties:

(i) $P\left(Z_{\infty}=z\right)=0$ for any $z \in(0,1)$.

(ii) If we have $P\left(\bigcap_{j=1}^{N}\left\{Z_{0, j}=0\right\}\right)+P\left(\bigcap_{j=1}^{N}\left\{Z_{0, j}=1\right\}\right)<1$, then $P\left(0<Z_{\infty}<1\right)>0$.

In particular, these facts entail that the asymptotic covariances in the following CLTs are "truly" random. Indeed, their random part $Z_{\infty}\left(1-Z_{\infty}\right)$ is different from zero with probability greater than zero and almost surely different from a constant in $(0,1)$.

Furthermore, it is interesting to note that the almost sure synchronization holds true without any assumptions on the initial configuration $\mathbf{Z}_{0}$ and for any choice of the weighted adjacency matrix $W$ with the required assumptions. Finally, note that the synchronization is induced along time independently of the fixed size $N$ of the network, and so it does not require a large-scale limit (i.e., the limit for $N \rightarrow+\infty$ ), which is usual in statistical mechanics for the study of interacting particle systems.

Regarding the convergence rate and the second-order asymptotic distribution of $\left(\mathbf{Y}_{n}-Z_{\infty} \mathbf{1}\right)$, setting for each $\gamma \in(1 / 2,1]$

$$
\begin{gathered}
\gamma_{0}:=\max \left\{\frac{1}{2}, 2 \gamma-1\right\} \in[1 / 2,1], \\
\widetilde{\Sigma}_{\gamma}:=\widetilde{\sigma}_{\gamma}^{2} \mathbf{1 1}{ }^{\top} \quad \text { with } \widetilde{\sigma}_{\gamma}^{2}:=\frac{\left\|\mathbf{v}_{1}\right\|^{2} c^{2}}{N(2 \gamma-1)}
\end{gathered}
$$


and

$$
\widetilde{U}=\left(\begin{array}{llll}
\mathbf{u}_{1} & \mathbf{u}_{2} & \ldots & \mathbf{u}_{N}
\end{array}\right)=\left(\begin{array}{ll}
N^{-1 / 2} \mathbf{1} & U
\end{array}\right) \quad \text { with } U:=\left(\begin{array}{lll}
\mathbf{u}_{2} & \ldots & \mathbf{u}_{N}
\end{array}\right),
$$

we obtain the following result.

Theorem 3.2. Under all the assumptions stated in Section 2, the following statements hold true:

(a) If $1 / 2<v<\gamma_{0}$, then

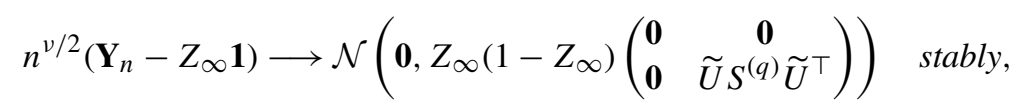

where, for $1 \leq j_{1}, j_{2} \leq N$,

$$
\left[S^{(q)}\right]_{j_{1} j_{2}}:=\frac{q}{2} \mathbf{v}_{j_{1}}^{\top} \mathbf{v}_{j_{2}}
$$

(b) If $\gamma_{0}<v<1$, then

$$
n^{\gamma-\frac{1}{2}}\left(\mathbf{Y}_{n}-Z_{\infty} \mathbf{1}\right) \longrightarrow \mathcal{N}\left(\mathbf{0}, Z_{\infty}\left(1-Z_{\infty}\right) \widetilde{\Sigma}_{\gamma}\right) \text { stably. }
$$

(c) If $v=\gamma_{0}<1$, then

$$
\begin{aligned}
& n^{\gamma-\frac{1}{2}}\left(\mathbf{Y}_{n}-Z_{\infty} \mathbf{1}\right)
\end{aligned}
$$

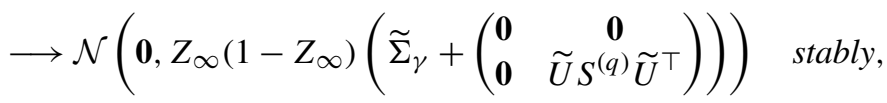

where $S^{(q)}$ is the same matrix defined in (a) by (3.8).

(d) If $v=\gamma_{0}=1$ (that is $\left.v=\gamma=1\right)$, then

$$
\begin{aligned}
& \sqrt{n}\left(\mathbf{Y}_{n}-Z_{\infty} \mathbf{1}\right) \\
& \quad \longrightarrow \mathcal{N}\left(\mathbf{0}, Z_{\infty}\left(1-Z_{\infty}\right)\left(\widetilde{\Sigma}_{1}+\left(\begin{array}{cc}
\widetilde{U} S^{11} \tilde{U}^{\top} & \widetilde{U} S^{12} \widetilde{U}^{\top} \\
\widetilde{U} S^{21} \widetilde{U}^{\top} & \widetilde{U} S^{22} \widetilde{U}^{\top}
\end{array}\right)\right) \quad\right. \text { stably, }
\end{aligned}
$$

where $S^{21}=\left(S^{12}\right)^{\top}$ and, for $2 \leq j_{1}, j_{2}, j \leq N$,

$$
\begin{aligned}
& {\left[S^{11}\right]_{11} }=\left[S^{11}\right]_{j_{1} 1}=\left[S^{11}\right]_{1 j_{2}}:=0, \quad\left[S^{11}\right]_{j_{1} j_{2}}:=\frac{c^{2}}{c\left(\alpha_{j_{1}}+\alpha_{j_{2}}\right)-1} \mathbf{v}_{j_{1}}^{\top} \mathbf{v}_{j_{2}}, \\
& {\left[S^{12}\right]_{11}=\left[S^{12}\right]_{1 j_{2}}:=0, \quad\left[S^{12}\right]_{j_{1} 1}:=\frac{c(q-c)}{c \alpha_{j_{1}}+q-1} \mathbf{v}_{j_{1}}^{\top} \mathbf{v}_{1}, } \\
& {\left[S^{12}\right]_{j_{1} j_{2}}:=\frac{c q\left(c \alpha_{j_{1}}+c-1\right)}{\left(c \alpha_{j_{1}}+c \alpha_{j_{2}}-1\right)\left(c \alpha_{j_{1}}+q-1\right)} \mathbf{v}_{j_{1}}^{\top} \mathbf{v}_{j_{2}}, } \\
& {\left[S^{22}\right]_{11}:=\frac{(q-c)^{2}}{2 q-1}\left\|\mathbf{v}_{1}\right\|^{2}, \quad\left[S^{22}\right]_{j 1}=\left[S^{22}\right]_{1 j}:=\frac{q(q-c)(c+q-1)}{\left(c \alpha_{j}+q-1\right)(2 q-1)} \mathbf{v}_{j}^{\top} \mathbf{v}_{1}, }
\end{aligned}
$$




$$
\begin{aligned}
{\left[S^{22}\right]_{j_{1} j_{2}}:=} & q^{2} \frac{c^{3}\left(\alpha_{j_{1}}+\alpha_{j_{2}}\right)+2 c^{2} q\left(\alpha_{j_{1}} \alpha_{j_{2}}+1\right)-c^{2}\left(\alpha_{j_{1}} \alpha_{j_{2}}+\alpha_{j_{1}}+\alpha_{j_{2}}+2\right)}{(2 q-1)\left(c\left(\alpha_{j_{1}}+\alpha_{j_{2}}\right)-1\right)\left(c \alpha_{j_{1}}+q-1\right)\left(c \alpha_{j_{2}}+q-1\right)} \mathbf{v}_{j_{1}}^{\top} \mathbf{v}_{j_{2}} \\
& +q^{2} \frac{c(q-1)^{2}\left(\alpha_{j_{1}}+\alpha_{j_{2}}\right)-(2 c+q-1)(q-1)}{(2 q-1)\left(c\left(\alpha_{j_{1}}+\alpha_{j_{2}}\right)-1\right)\left(c \alpha_{j_{1}}+q-1\right)\left(c \alpha_{j_{2}}+q-1\right)} \mathbf{v}_{j_{1}}^{\top} \mathbf{v}_{j_{2}} .
\end{aligned}
$$

(e) If $\gamma_{0}<v=1$, then

$$
\begin{aligned}
& n^{\gamma-\frac{1}{2}}\left(\mathbf{Y}_{n}-Z_{\infty} \mathbf{1}\right) \\
& \quad \longrightarrow \mathcal{N}\left(\mathbf{0}, Z_{\infty}\left(1-Z_{\infty}\right)\left(\widetilde{\Sigma}_{\gamma}+\frac{\left\|\mathbf{v}_{1}\right\|^{2} c^{2}}{N[2 q-(2 \gamma-1)]}\left(\begin{array}{cc}
\mathbf{0} & \mathbf{0} \\
\mathbf{0} & \mathbf{1 1}^{\top}
\end{array}\right)\right)\right) \text { stably. }
\end{aligned}
$$

Remark 3.1. Looking at the asymptotic covariance matrices in the different cases of the above theorem, note that in case (a) the convergence rate of the first component is bigger than the one of the second component. Indeed, from our previous work Aletti, Crimaldi and Ghiglietti [1], we know that it is $n^{\gamma_{0} / 2}$. On the other hand, there are cases (see (b), (c) and (e)) in which the convergence rates of the two components are the same, although the discount factors $r_{n} \sim c n^{-\gamma}$ and $q_{n, n} \sim q n^{-v}$ in (1.9) have different convergence rates.

Remark 3.2. Recall that we have $1 \leq 1+\left\|\mathbf{v}_{1}-\mathbf{u}_{1}\right\|^{2}=\left\|\mathbf{v}_{1}\right\|^{2} \leq N$. Therefore we obtain the following lower and upper bounds (that do not depend on $W$ ) for $\widetilde{\sigma}_{\gamma}^{2}$ and for the second term in the asymptotic covariance of relation (3.12):

$$
\begin{aligned}
\frac{c^{2}}{N(2 \gamma-1)} & \leq \tilde{\sigma}_{\gamma}^{2} \leq \frac{c^{2}}{2 \gamma-1} \quad \text { and } \\
\frac{c^{2}}{N[2 q-(2 \gamma-1)]} & \leq \frac{\left\|\mathbf{v}_{1}\right\|^{2} c^{2}}{N[2 q-(2 \gamma-1)]} \leq \frac{c^{2}}{2 q-(2 \gamma-1)} .
\end{aligned}
$$

Notice that the lower bound is achieved when $\mathbf{v}_{1}=\mathbf{u}_{1}=N^{-1 / 2} \mathbf{1}$, that is, when $W$ is doubly stochastic, which means $W \mathbf{1}=W^{\top} \mathbf{1}=\mathbf{1}$.

Remark 3.3. The main goal of this work is to provide results for a system of $N \geq 2$ interacting reinforced stochastic processes. However, it is worth to note that Theorem 3.1, and the consequent limit (3.1), hold true also for $N=1$. Moreover, statements (d) and (e) of Theorem 3.2 with $N=1$ are true. Finally, statements (a), (b) and (c) of Theorem 3.2 with $N=1$ (and so without the condition on $\lambda^{*}$ ) can be proven with the same proof provided in the sequel (see the following Remark 5.2).

We conclude this section with the example of the "mean-field" interaction, which is a simple, but widely used in applications (for instance, in Game Theory), type of interacting mechanism. Other examples of interaction can be found in Aletti, Crimaldi and Ghiglietti [1]: precisely, the case of a "cycle-interaction", where the vertex form a circle because each vertex $h$, with $h=$ $1, \ldots, N-1$ influences only the vertex $h+1$ and the vertex $N$ influences only the vertex 1 , and 
the case in which there exists a "special vertex" whose influence on the vertices of the graph is different with respect to the one of all the others.

Example 3.1. The mean-field interaction can be expressed in terms of a particular weighted adjacency matrix $W$ as follows: for any $1 \leq j_{1}, j_{2} \leq N$

$$
w_{j_{1}, j_{2}}=\frac{\alpha}{N}+(1-\alpha) \delta_{j_{1}, j_{2}} \quad \text { with } \alpha \in[0,1],
$$

where $\delta_{j_{1}, j_{2}}$ is equal to 1 when $j_{1}=j_{2}$ and to 0 otherwise. Note that $W$ in (3.13) is irreducible for $\alpha>0$ and so we are going to consider this case. Since $W$ is doubly stochastic, we have $\mathbf{v}_{1}=\mathbf{u}_{1}=N^{-1 / 2} \mathbf{1}$. Moreover, since $W$ is also symmetric, we have $\widetilde{U}=\widetilde{V}$ and so $\widetilde{U} \widetilde{U}^{\top}=I$ and $\widetilde{V}^{\top} \widetilde{V}=I$. Finally, we have $\lambda_{j}=1-\alpha$ for all $j \geq 2$ and, consequently, we obtain

$$
\begin{gathered}
S^{(q)}=\frac{q}{2} I, \quad\left\{\left[S^{11}\right]_{j_{1} j_{2}}: 2 \leq j_{1}, j_{2} \leq N\right\}=\frac{c^{2}}{2 c \alpha-1} I, \\
{\left[S^{12}\right]_{j_{1} 1}=0 \quad \text { for } 2 \leq j_{1} \leq N, \quad\left\{\left[S^{12}\right]_{j_{1} j_{2}}: 2 \leq j_{1}, j_{2} \leq N\right\}=\frac{q c(c \alpha+c-1)}{(2 c \alpha-1)(c \alpha+q-1)} I,} \\
{\left[S^{22}\right]_{11}=\frac{(q-c)^{2}}{2 q-1}, \quad\left[S^{22}\right]_{j 1}=\left[S_{1 j}^{22}\right]=0 \quad \text { for } 2 \leq j \leq N,} \\
\left\{\left[S^{22}\right]_{j_{1} j_{2}}: 2 \leq j_{1}, j_{2} \leq N\right\} \\
=\frac{(q c)^{2}\left[\left(\alpha^{2}+1\right)(2 q-1)+2 \alpha(c-1)-1+\left(2 \alpha-c^{-1}\right)(q-1)^{2}-2 c^{-1}(q-1)\right]}{(2 q-1)(2 c \alpha-1)(c \alpha+q-1)^{2}} I,
\end{gathered}
$$

and the condition $\operatorname{Re}\left(\lambda^{*}\right)<1-(2 c)^{-1}$ when $\gamma=1$ becomes $2 c \alpha>1$.

\section{Useful results for statistical applications}

The first convergence result provided in this section can be used for the construction of asymptotic confidence intervals for the limit random variable $Z_{\infty}$, that requires the observation of the actions $X_{n, j}$ adopted by the agents and the knowledge of the following parameters:

- $N$ : the number of agents in the network;

- $\mathbf{v}_{1}$ : the right eigenvector of $W$ associated to $\lambda_{1}=1$ (note that it is not required to know the whole weighted adjacency matrix $W$, for example, we have $\mathbf{v}_{1}=\mathbf{u}_{1}=N^{-1 / 2} \mathbf{1}$ for any doubly stochastic matrix);

- $\gamma$ and $c$ : the parameters that describe the first-order asymptotic approximation of the sequence $\left(r_{n}\right)_{n}$;

- $v$ and $q$ : the parameters that describe the first-order asymptotic approximation of the sequence $\left(q_{n, n}\right)_{n}$ (recall that the weights $q_{n, k}$ are chosen and so $v$ and $q$ are always known and, sometimes, they can be optimally chosen). 
We point out that it is not required the observation of the random variables $Z_{n, j}$, nor the knowledge of the initial random variables $\left\{Z_{0, j}: j \in V\right\}$ and nor of the exact expression of the sequence $\left(r_{n}\right)_{n}$.

The second result stated in this section can be employed for the construction of asymptotic critical regions for statistical tests on the weighted adjacency matrix $W$ based on the weighted empirical means of the agents' actions (given the values of $\gamma, v, c, q$ and $N$ ). See Remark 4.2 for the details. In particular, we point out that in our previous work Aletti, Crimaldi and Ghiglietti [2] we succeeded to provide a testing procedure based on the standard empirical means only for the case $\gamma=1$; while we announced further future investigation for the case $1 / 2<\gamma<1$. In the present work, we face and solve this issue, providing a test statistics for all the values of the parameters. Indeed the following Theorem 4.2 covers all the cases for the pair $(\gamma, v)$.

Let us consider the decomposition $\mathbf{N}_{n}=\mathbf{1} \widetilde{N}_{n}+\mathbf{N}_{n}^{\prime}$, where

$$
\mathbf{1} \widetilde{N}_{n}:=\mathbf{u}_{1} \mathbf{v}_{1}^{\top} \mathbf{N}_{n}=N^{-1 / 2} \mathbf{1} \mathbf{v}_{1}^{\top} \mathbf{N}_{n} \quad \text { and } \quad \mathbf{N}_{n}^{\prime}:=\mathbf{N}_{n}-\mathbf{1} \tilde{N}_{n}=\left(I-\mathbf{u}_{1} \mathbf{v}_{1}^{\top}\right) \mathbf{N}_{n} .
$$

Concerning the first term, by (2.2) and the almost sure synchronization (3.1), we immediately obtain $\tilde{N}_{n} \stackrel{\text { a.s. }}{\longrightarrow} Z_{\infty}$. Moreover, under all the assumptions stated in Section 2, setting

$$
\widetilde{\sigma}^{2}:=\frac{\left\|\mathbf{v}_{1}\right\|^{2}}{N} \times \begin{cases}\frac{q}{2} & \text { if } v<\gamma_{0} \text { or } v=\gamma_{0}<1, \\ \frac{(q-c)^{2}}{2 q-1} & \text { if } v=\gamma_{0}=1(\text { that is } v=\gamma=1), \\ \frac{c^{2}}{2 q-(2 \gamma-1)} & \text { if } \gamma_{0}<v=1,\end{cases}
$$

where $\gamma_{0}$ and $\tilde{\sigma}_{\gamma}^{2}$ are defined in (3.4) and in (3.5), respectively, we have the following result:

Theorem 4.1. Under all the assumptions stated in Section 2, the following statements hold true:

(a) If $v<\gamma_{0}$, then

$$
n^{\nu / 2}\left(\widetilde{N}_{n}-Z_{\infty}\right) \longrightarrow \mathcal{N}\left(0, Z_{\infty}\left(1-Z_{\infty}\right) \widetilde{\sigma}^{2}\right) \text { stably. }
$$

(b) If $\gamma_{0}<v<1$, then

$$
n^{\gamma-\frac{1}{2}}\left(\widetilde{N}_{n}-Z_{\infty}\right) \longrightarrow \mathcal{N}\left(0, Z_{\infty}\left(1-Z_{\infty}\right) \tilde{\sigma}_{\gamma}^{2}\right) \text { stably. }
$$

(c) If $v=\gamma_{0}$ or $v=1$ (i.e. $v=\gamma_{0}<1$ or $v=\gamma_{0}=1$ or $\left.\gamma_{0}<v=1\right)$, then

$$
n^{\gamma-\frac{1}{2}}\left(\widetilde{N}_{n}-Z_{\infty}\right) \longrightarrow \mathcal{N}\left(0, Z_{\infty}\left(1-Z_{\infty}\right)\left(\widetilde{\sigma}_{\gamma}^{2}+\tilde{\sigma}^{2}\right)\right) \text { stably }
$$

Note that $\widetilde{\sigma}^{2}$ has not been defined in the case $\gamma_{0}<v<1$, i.e. in the case (b) of the above result, because in this case it does not appear in the asymptotic covariance matrix. In the following remark, we briefly describe how to construct asymptotic confidence intervals for $Z_{\infty}$ based on the random variable $\widetilde{N}_{n}$ and we point out the advantages of employing the weighted empirical means with $v<1$, instead of the simple empirical means (for which we have $v=q=1$ ), providing a short discussion on the possible "optimal choice" of $v$ and $q$ : 
Remark 4.1. If we define $v^{2}$ equal to $\tilde{\sigma}^{2}$, or $\tilde{\sigma}_{\gamma}^{2}$, or $\left(\tilde{\sigma}_{\gamma}^{2}+\tilde{\sigma}^{2}\right)$ and $e$ equal to $v / 2$ or $\gamma-\frac{1}{2}$, according to the values of the pair $(\gamma, \nu)$, so that, by Theorem 4.1 , we have the stable convergence of $n^{e}\left(\widetilde{N}_{n}-Z_{\infty}\right)$ to $\mathcal{N}\left(0, Z_{\infty}\left(1-Z_{\infty}\right) v^{2}\right)$, then, employing $\widetilde{N}_{n}$ as a strong consistent estimator of $Z_{\infty}$, we get (since, as recalled in Appendix A, stable convergence and convergence in probability combine well) $n^{e}\left(\widetilde{N}_{n}-Z_{\infty}\right) / \sqrt{v^{2} \widetilde{N}_{n}\left(1-\widetilde{N}_{n}\right)}$ convergent to $\mathcal{N}(0,1)$. Hence, if $z_{\theta}$ is the quantile of the standard normal distribution of order $1-\theta / 2$, then $\mathrm{CI}_{1-\theta}\left(Z_{\infty}\right)=\widetilde{N}_{n} \pm \frac{z_{\theta}}{n^{e}} v \sqrt{\widetilde{N}_{n}\left(1-\widetilde{N}_{n}\right)}$ provides an asymptotic confidence interval for $Z_{\infty}$, with (approximate) level $(1-\theta)$.

The convergence rates and the asymptotic variances expressed in the cases of Theorem 4.1 allows us to make some considerations on the existence of an "optimal" choice of the parameters $v$ and $q$ in order to "maximize the convergence" of $\widetilde{N}_{n}$ towards the random limit $Z_{\infty}$. Indeed, first note that the convergence rate in case (a) is slower than the rates of the other two cases, and, moreover, the asymptotic variance in case (c) is strictly larger than the variance in case (b). Hence, the interval $\gamma_{0}<v<1$ in case (b) provides an "optimal" range of values where the parameter $v$ should be chosen. In addition, looking into the proof of Theorem 4.1 , it is possible to investigate more deeply into the behavior of $\widetilde{N}_{n}$ and so derive more accurate optimality conditions on the values of $v$ and $q$ (see the following Remark 6.2).

Analogously, concerning the term $\mathbf{N}_{n}^{\prime}=\left(I-\mathbf{u}_{1} \mathbf{v}_{1}^{\top}\right) \mathbf{N}_{n}$, from (2.2) and the almost sure synchronization (3.1), we obtain $\mathbf{N}_{n}^{\prime} \stackrel{\text { a.s. }}{\rightarrow}$ 0. Moreover, setting

$$
\widetilde{U}_{-1}:=\left(\begin{array}{llll}
\mathbf{0} & \mathbf{u}_{2} & \ldots & \mathbf{u}_{N}
\end{array}\right)=\left(\begin{array}{ll}
\mathbf{0} & U
\end{array}\right),
$$

we get the following theorem.

Theorem 4.2. Under all the assumptions stated in Section 2, the following statements hold true:

(a) If $v<\gamma$, then

$$
n^{\frac{\nu}{2}} \mathbf{N}_{n}^{\prime} \longrightarrow \mathcal{N}\left(\mathbf{0}, Z_{\infty}\left(1-Z_{\infty}\right) \widetilde{U}_{-1} S^{(q)} \tilde{U}_{-1}^{\top}\right) \text { stably }
$$

where $S^{(q)}$ is defined in (3.8).

(b) If $v=\gamma$, then

$$
n^{\frac{\nu}{2}} \mathbf{N}_{n}^{\prime} \longrightarrow \mathcal{N}\left(\mathbf{0}, Z_{\infty}\left(1-Z_{\infty}\right) \widetilde{U}_{-1} S_{\gamma}^{22} \widetilde{U}_{-1}^{\top}\right) \text { stably }
$$

where, for any $2 \leq j_{1}, j_{2} \leq N$, we have that $\left[S_{\gamma}^{22}\right]_{11},\left[S_{\gamma}^{22}\right]_{1 j_{2}}$ and $\left[S_{\gamma}^{22}\right]_{j_{1} 1}$ are not needed to be defined since the first column of $\widetilde{U}_{-1}$ is $\mathbf{0}$, while the remaining elements $\left[S_{\gamma}^{22}\right]_{j_{1} j_{2}}$ are defined as

$$
\begin{aligned}
& q^{2} \frac{c^{3}\left(\alpha_{j_{1}}+\alpha_{j_{2}}\right)+2 c^{2} q\left(\alpha_{j_{1}} \alpha_{j_{2}}+1\right)-\mathbb{1}_{\{\gamma=1\}} c^{2}\left(\alpha_{j_{1}} \alpha_{j_{2}}+\alpha_{j_{1}}+\alpha_{j_{2}}+2\right)}{\left(2 q-\mathbb{1}_{\{\gamma=1\}}\right)\left(c\left(\alpha_{j_{1}}+\alpha_{j_{2}}\right)-\mathbb{1}_{\{\gamma=1\}}\right)\left(c \alpha_{j_{1}}+q-\mathbb{1}_{\{\gamma=1\}}\right)\left(c \alpha_{j_{2}}+q-\mathbb{1}_{\{\gamma=1\}}\right)} \mathbf{v}_{j_{1}}^{\top} \mathbf{v}_{j_{2}} \\
& \quad+q^{2} \frac{c\left(q-\mathbb{1}_{\{\gamma=1\}}\right)^{2}\left(\alpha_{j_{1}}+\alpha_{j_{2}}\right)-\mathbb{1}_{\{\gamma=1\}}(2 c+q-1)(q-1)}{\left(2 q-\mathbb{1}_{\{\gamma=1\}}\right)\left(c\left(\alpha_{j_{1}}+\alpha_{j_{2}}\right)-\mathbb{1}_{\{\gamma=1\}}\right)\left(c \alpha_{j_{1}}+q-\mathbb{1}_{\{\gamma=1\}}\right)\left(c \alpha_{j_{2}}+q-\mathbb{1}_{\{\gamma=1\}}\right)} \\
& \quad \times \mathbf{v}_{j_{1}}^{\top} \mathbf{v}_{j_{2}} .
\end{aligned}
$$


(c) If $\gamma<v$, then

$$
n^{\frac{v}{2}} \mathbf{N}_{n}^{\prime} \longrightarrow \mathcal{N}\left(\mathbf{0}, Z_{\infty}\left(1-Z_{\infty}\right) \widetilde{U}_{-1} S \tilde{U}_{-1}^{\top}\right) \text { stably, }
$$

where, for any $2 \leq j_{1}, j_{2} \leq N$, we have that $[S]_{11},[S]_{1 j_{2}}$ and $[S]_{j_{1} 1}$ are not needed to be defined since the first column of $\widetilde{U}_{-1}$ is $\mathbf{0}$, while the remaining elements $[S]_{j_{1} j_{2}}$ are defined as

$$
\begin{aligned}
& q^{2}\left(\left(\frac{\lambda_{j_{1}} \lambda_{j_{2}}}{\alpha_{j_{1}} \alpha_{j_{2}}}\right) \frac{1}{2 q-\mathbb{1}_{\{v=1\}}(2 \gamma-1)}+\left(\frac{\lambda_{j_{1}}}{\alpha_{j_{1}}}+\frac{\lambda_{j_{2}}}{\alpha_{j_{2}}}\right) \frac{1}{2 q-\mathbb{1}_{\{\nu=1\}} \gamma}+\frac{1}{2 q-\mathbb{1}_{\{\nu=1\}}}\right) \\
& \quad \times \mathbf{v}_{j_{1}}^{\top} \mathbf{v}_{j_{2}} .
\end{aligned}
$$

Note that the convergence rate for $\left(\mathbf{N}_{n}^{\prime}\right)$ is always $n^{\nu / 2}$. In the following remark, we briefly describe how to use $\widetilde{N}_{n}$ and $\mathbf{N}_{n}^{\prime}$ in order to perform a test on the matrix $W$ (for any values of $\gamma$ and $v)$.

Remark 4.2. Theorem 4.2 can be used for testing the hypothesis that the network is characterized by a given weighted adjacency matrix $W_{0}$, that is, $H_{0}: W=W_{0}$, using the random variables $\mathbf{N}_{n}^{\prime}$ and $\widetilde{N}_{n}$, observed at the vertices (the parameters $\gamma, v, c, q$ and $N$ are supposed known). Indeed, fixed the weighted adjacency matrix assumed under $H_{0}$, that is, $W_{0}$, we can compute for it the vectors $\mathbf{u}_{n}, \mathbf{v}_{n}$ and the eigenvalues $\lambda_{j}$, and hence $\widetilde{N}_{n}$ and $\mathbf{N}_{n}^{\prime}$, and, according to the value of $v$ and $\gamma$, the matrix, say $M$, specified in Theorem 4.2 so that $n^{\frac{v}{2}} \mathbf{N}_{n}^{\prime} \stackrel{d}{\sim} \mathcal{N}\left(\mathbf{0}, Z_{\infty}\left(1-Z_{\infty}\right) M\right)$. If $L$ is such that $\mathrm{LML}^{\top}=I$ and we employ $\widetilde{N}_{n}$ as a strong consistent estimator of $Z_{\infty}$, then, under $H_{0}$, we get (since, as recalled in Appendix A, stable convergence and convergence in probability combine well) $\frac{n^{\frac{v}{2}}}{\sqrt{\widetilde{N}_{n}\left(1-\widetilde{N}_{n}\right)}} L \mathbf{N}_{n}^{\prime} \stackrel{d}{\sim} \mathcal{N}(\mathbf{0}, I)$ and so $\frac{n^{\nu}}{N_{n}\left(1-\widetilde{N}_{n}\right)}\left(\mathbf{N}_{n}^{\prime}\right)^{\top} L^{\top} L \mathbf{N}_{n}^{\prime} \stackrel{d}{\sim} \chi_{N-1}^{2}$. We may use this asymptotic distribution in order to construct asymptotic critical regions for testing $H_{0}$. The performance in terms of power of this testing procedure is strongly related to the considered adjacency matrix $W_{1}$ belonging to the alternative hypothesis $H_{1}$. See Aletti, Crimaldi and Ghiglietti $[1,2]$ for a discussion of this issue.

In the following example we go on with the analysis of the mean-field interaction, providing a simple application of the general testing procedure described in Remark 4.2.

Example 4.1. If we consider again the mean-field interaction (see (3.13)), we have $\mathbf{N}_{n}^{\prime}=(I-$ $\left.N^{-1} \mathbf{1 1}^{\top}\right) \mathbf{N}_{n}$ (because $\mathbf{v}_{1}=\mathbf{u}_{1}=N^{-1 / 2} \mathbf{1}$ ). Moreover, since $\widetilde{U}=\widetilde{V}$ and so $\widetilde{V}^{\top} \widetilde{V}=I$, we find $S^{(q)}=\frac{q}{2} I$,

$$
\left\{\left[S_{\gamma}^{22}\right]_{j_{1} j_{2}}: 2 \leq j_{1}, j_{2} \leq N\right\}=s_{\gamma}^{22} I
$$

with

$$
\begin{aligned}
s_{\gamma}^{22}:= & \left(q ^ { 2 } \left[c^{2}\left(\alpha^{2}+1\right)\left(2 q-\mathbb{1}_{\{\gamma=1\}}\right)+2 c^{2} \alpha\left(c-\mathbb{1}_{\{\gamma=1\}}\right)-\mathbb{1}_{\{\gamma=1\}} c^{2}\right.\right. \\
& \left.\left.+2 \alpha c\left(q-\mathbb{1}_{\{\gamma=1\}}\right)^{2}-\mathbb{1}_{\{\gamma=1\}}(2 c+q-1)(q-1)\right]\right) \\
& /\left(\left(2 q-\mathbb{1}_{\{\gamma=1\}}\right)\left(2 c \alpha-\mathbb{1}_{\{\gamma=1\}}\right)\left(c \alpha+q-\mathbb{1}_{\{\gamma=1\}}\right)^{2}\right)
\end{aligned}
$$


and

$$
\begin{aligned}
& S=s I \text { with } \\
& s:=q^{2}\left(\left(\frac{1-\alpha}{\alpha}\right)^{2} \frac{1}{2 q-\mathbb{1}_{\{\nu=1\}}(2 \gamma-1)}+2 \frac{(1-\alpha)}{\alpha} \frac{1}{2 q-\mathbb{1}_{\{\nu=1\}} \gamma}+\frac{1}{2 q-\mathbb{1}_{\{\nu=1\}}}\right) .
\end{aligned}
$$

Hence, since $\widetilde{U}_{-1} I \widetilde{U}_{-1}^{\top}=U U^{\top}=I-N^{-1} \mathbf{1 1}{ }^{\top}$, we get that

$$
n^{\nu / 2}\left(I-N^{-1} \mathbf{1 1}^{\top}\right) \mathbf{N}_{n} \longrightarrow \mathcal{N}\left(0, Z_{\infty}\left(1-Z_{\infty}\right) s^{*}\left(I-N^{-1} \mathbf{1 1}^{\top}\right)\right) \quad \text { stably }
$$

where $s^{*}$ is equal to $q / 2$ or $s_{\gamma}^{22}$ or $s$, according to the values of $v$ and $\gamma$. Finally, using the relations $U^{\top} U=I$ and $U U^{\top}=I-N^{-1} \mathbf{1 1}^{\top}$ and employing $\widetilde{N}_{n}$ as a strong consistent estimator of $Z_{\infty}$, we get (write $\mathbf{N}_{n}^{\prime}$ as $U U^{\top} \mathbf{N}_{n}$ and take $M=s^{*}\left(I-N^{-1} \mathbf{1 1}^{\top}\right)=s^{*} U U^{\top}$ and $L=U^{\top} / \sqrt{s^{*}}$ in the general procedure described in Remark 4.2)

$$
\frac{n^{\nu / 2}}{\sqrt{\widetilde{N}_{n}\left(1-\widetilde{N}_{n}\right) s^{*}}} U^{\top} \mathbf{N}_{n} \stackrel{d}{\sim} \mathcal{N}(0, I) \quad \text { and } \quad \frac{n^{\nu}}{\widetilde{N}_{n}\left(1-\widetilde{N}_{n}\right) s^{*}} \mathbf{N}_{n}^{\top}\left(I-N^{-1} \mathbf{1 1}^{\top}\right) \mathbf{N}_{n} \stackrel{d}{\sim} \chi_{N-1}^{2}
$$

Given the values of $\gamma, v, c, q$ and $N$, this last asymptotic distribution can be used in order to perform a statistical test on the parameter $\alpha$ in the definition of $W$ (see (3.13)), that is with $H_{0}: W=W_{0}$, where $W_{0}$ is the matrix corresponding to a certain value of $\alpha$.

\section{Proof of the results on the joint stochastic process}

Here we prove the convergence results stated in Section 3.

\subsection{Proof of Theorem 3.1}

As already recalled (see (3.2)), we have $\mathbf{Z}_{n} \stackrel{\text { a.s. }}{\rightarrow} Z_{\infty}$. Hence, since the condition $W^{\top} \mathbf{1}=\mathbf{1}$ and the equality (1.8), we get $E\left[\mathbf{X}_{n} \mid \mathcal{F}_{n-1}\right] \stackrel{\text { a.s. }}{\rightarrow} Z_{\infty} \mathbf{1}$. Therefore, the convergence $\mathbf{N}_{n} \stackrel{\text { a.s. }}{\rightarrow} Z_{\infty} \mathbf{1}$ follows from (Aletti, Crimaldi and Ghiglietti [2], Lemma B.1) with $c_{k}=k^{v}, v_{n, k}=c_{k} q_{n, k}$ and $\eta=1$. Note that the assumptions on the weights $q_{n, k}=a_{k} / \sum_{l=1}^{n} a_{l}$, easily implies that $c_{k}$ and $v_{n, k}$ satisfy the conditions required in the employed lemma: indeed, by definition, we have $\sum_{k=1}^{n} q_{n, k}=1$ and from the second relation in (2.4) we get $\sum_{n=1}^{+\infty} a_{n}=+\infty$ and

$$
n^{v} a_{n}=q \sum_{l=1}^{n} a_{l}+O\left(n^{-v} \sum_{l=1}^{n} a_{l}\right)=q \sum_{l=1}^{n} a_{l}+O\left(a_{n}\left(n^{v} q_{n, n}\right)^{-1}\right)=q \sum_{l=1}^{n} a_{l}+O\left(a_{n}\right)
$$


and so we obtain

$$
\begin{aligned}
\lim _{n} v_{n, k} & =c_{k} a_{k} \lim _{n} \frac{1}{\sum_{l=1}^{n} a_{l}}=0, \\
\lim _{n} v_{n, n} & =\lim _{n} c_{n} q_{n, n}=q, \\
\lim _{n} \sum_{k=1}^{n} \frac{v_{n, k}}{c_{k}} & =\lim _{n} \sum_{k=1}^{n} q_{n, k}=1
\end{aligned}
$$

and

$$
\begin{aligned}
\sum_{k=1}^{n}\left|v_{n, k}-v_{n, k-1}\right| & =\frac{1}{\sum_{l=1}^{n} a_{l}} \sum_{k=1}^{n} k^{v} a_{k}-(k-1)^{v} a_{k-1} \\
& =\frac{1}{\sum_{l=1}^{n} a_{l}}\left[\sum_{k=1}^{n} q\left(\sum_{l=1}^{k} a_{l}-\sum_{l=1}^{k-1} a_{l}\right)+O\left(\sum_{k=1}^{n} a_{k}\right)\right] \\
& =q \frac{\sum_{k=1}^{n} a_{k}}{\sum_{l=1}^{n} a_{l}}+O(1)=O(1) .
\end{aligned}
$$

\subsection{Decomposition of the joint stochastic process}

In this section, we describe the main tool used in the following proofs, that is a suitable decomposition of the joint stochastic process $\mathbf{Y}:=\left(\mathbf{Y}_{n}\right)_{n}$. Indeed, in order to determine the convergence rate and the second-order asymptotic distribution of $\left(\mathbf{Y}_{n}-Z_{\infty} \mathbf{1}\right)$ for any values of the parameters, we need to decompose $\mathbf{Y}$ into a sum of "primitive" stochastic processes, and then establish the asymptotic behavior for each one of them. As we will see, they converge at different rates.

Let us express the dynamics (1.9) of the stochastic processes $\left(\mathbf{Z}_{n}\right)_{n}$ and $\left(\mathbf{N}_{n}\right)_{n}$ as follows:

$$
\left\{\begin{array}{l}
\mathbf{Z}_{n}-\mathbf{Z}_{n-1}=-\widehat{r}_{n-1}\left(I-W^{\top}\right) \mathbf{Z}_{n-1}+\widehat{r}_{n-1} \Delta \mathbf{M}_{n}+\Delta \mathbf{R}_{Z, n}, \\
\mathbf{N}_{n}-\mathbf{N}_{n-1}=-\widehat{q}_{n, n}\left(\mathbf{N}_{n-1}-W^{\top} \mathbf{Z}_{n-1}\right)+\widehat{q}_{n, n} \Delta \mathbf{M}_{n}+\Delta \mathbf{R}_{N, n},
\end{array}\right.
$$

where $\Delta \mathbf{M}_{n}:=\left(\mathbf{X}_{n}-W^{\top} \mathbf{Z}_{n-1}\right)$ is a martingale increment with respect to the filtration $\mathcal{F}:=$ $\left(\mathcal{F}_{n}\right)_{n}$, while $\Delta \mathbf{R}_{Z, n}:=\left(r_{n-1}-\widehat{r}_{n-1}\right)\left(\mathbf{X}_{n}-\mathbf{Z}_{n-1}\right)$ and $\Delta \mathbf{R}_{N, n}:=\left(q_{n, n}-\widehat{q}_{n, n}\right)\left(\mathbf{X}_{n}-\mathbf{Z}_{n-1}\right)$ are two remainder terms. Hence, by means of (5.1), the dynamics of the stochastic process $\mathbf{Y}$ can be expressed as

$$
\mathbf{Y}_{n}=\left(I-Q_{n}\right) \mathbf{Y}_{n-1}+R_{n} \Delta \mathbf{M}_{Y, n}+\Delta \mathbf{R}_{Y, n},
$$

where $\Delta \mathbf{M}_{Y, n}:=\left(\Delta \mathbf{M}_{n}, \Delta \mathbf{M}_{n}\right)^{\top}, \Delta \mathbf{R}_{Y, n}:=\left(\Delta \mathbf{R}_{Z, n}, \Delta \mathbf{R}_{N, n}\right)^{\top}$,

$$
Q_{n}:=\left(\begin{array}{cc}
\widehat{r}_{n-1}\left(I-W^{\top}\right) & \mathbf{0} \\
-\widehat{q}_{n, n} W^{\top} & \widehat{q}_{n, n} I
\end{array}\right) \quad \text { and } \quad R_{n}:=\left(\begin{array}{cc}
\widehat{r}_{n-1} I & \mathbf{0} \\
\mathbf{0} & \widehat{q}_{n, n} I
\end{array}\right)
$$


Now, we want to decompose the stochastic process $\mathbf{Y}$ in a sum of stochastic processes, whose dynamics are of the same types of (5.2), but more tractable. To this purpose, we define, for each $j=$ $1, \ldots, N$, two vectors $\mathbf{u}_{j(1)}$ and $\mathbf{u}_{j(2)}$ of dimension $2 N$ as follows. We set $U_{j}:=\left(\mathbf{u}_{j(1)}, \mathbf{u}_{j(2)}\right)$, that is the matrix of dimension $2 N \times 2$ having $\mathbf{u}_{j(1)}$ and $\mathbf{u}_{j(2)}$ as columns, and we impose the following relations:

$$
U_{j}=U_{j}^{*} P_{j} \quad \text { with } U_{j}^{*}:=\left(\begin{array}{cc}
\mathbf{u}_{j} & \mathbf{0} \\
\mathbf{0} & \mathbf{u}_{j}
\end{array}\right) \quad \text { and } \quad P_{j}:=\left(\begin{array}{cc}
1 & 0 \\
g\left(\lambda_{j}\right) & 1
\end{array}\right),
$$

and, for any $n \geq 1$,

$$
Q_{n} U_{j}=U_{j} D_{Q, j, n}, \quad \text { where } D_{Q, j, n}:=\left(\begin{array}{cc}
\widehat{r}_{n-1}\left(1-\lambda_{j}\right) & 0 \\
-\lambda_{j} h_{n}\left(\lambda_{j}\right) & \widehat{q}_{n, n}
\end{array}\right) .
$$

We recall that $\lambda_{j}$ and $\mathbf{u}_{j}$ denote the eigenvalues and the left eigenvectors of $W$, respectively. The above functions $g$ and $h_{n}$ will be suitable defined later on. In particular, we will define $h_{n}$ in such a way that the sequence $\left(h_{n}\left(\lambda_{j}\right)\right)_{n}$ converges to zero at the biggest possible rate. In order to solve the above system of equations, we firstly observe that, by (5.4), we have

$$
\begin{aligned}
\mathbf{u}_{j(1)} & =\left(\begin{array}{c}
\mathbf{u}_{j} \\
g\left(\lambda_{j}\right) \mathbf{u}_{j}
\end{array}\right), \quad \mathbf{u}_{j(2)}=\left(\begin{array}{c}
\mathbf{0} \\
\mathbf{u}_{j}
\end{array}\right), \\
Q_{n} U_{j} & =Q_{n} U_{j}^{*} P_{j} \\
& =U_{j}^{*}\left(\begin{array}{cc}
\widehat{r}_{n-1}\left(1-\lambda_{j}\right) & 0 \\
-\widehat{q}_{n, n} \lambda_{j} & \widehat{q}_{n, n}
\end{array}\right) P_{j} \\
& =U_{j}^{*}\left(\begin{array}{cc}
\widehat{r}_{n-1}\left(1-\lambda_{j}\right) & 0 \\
-\widehat{q}_{n, n} \lambda_{j}+\widehat{q}_{n, n} g\left(\lambda_{j}\right) & \widehat{q}_{n, n}
\end{array}\right)
\end{aligned}
$$

and

$$
U_{j} D_{Q, j, n}=U_{j}^{*} P_{j} D_{Q, j, n}=U_{j}^{*}\left(\begin{array}{cc}
\widehat{r}_{n-1}\left(1-\lambda_{j}\right) & 0 \\
\widehat{r}_{n-1}\left(1-\lambda_{j}\right) g\left(\lambda_{j}\right)-\lambda_{j} h_{n}\left(\lambda_{j}\right) & \widehat{q}_{n, n}
\end{array}\right) .
$$

Then, combining together (5.7) and (5.8) in order to satisfy (5.5), we obtain

$$
-\widehat{q}_{n, n} \lambda_{j}+\widehat{q}_{n, n} g\left(\lambda_{j}\right)=\widehat{r}_{n-1}\left(1-\lambda_{j}\right) g\left(\lambda_{j}\right)-\lambda_{j} h_{n}\left(\lambda_{j}\right)
$$

from which we get the equality

$$
\lambda_{j}\left[\widehat{q}_{n, n}-h_{n}\left(\lambda_{j}\right)\right]=g\left(\lambda_{j}\right)\left[\widehat{q}_{n, n}-\widehat{r}_{n-1}\left(1-\lambda_{j}\right)\right] .
$$

Now, for all values of $\gamma, v$ and $j \in\{1, \ldots, N\}$, we want to define $g\left(\lambda_{j}\right)$ and $h_{n}\left(\lambda_{j}\right)$ in such a way that (5.9) is verified for any $n$ and $h_{n}\left(\lambda_{j}\right)$ vanishes to zero with the biggest possible rate. To this end, we note that by (5.9) we have the following facts:

- if $j=1$, then $\lambda_{1}=1$, and we can set $h_{n}\left(\lambda_{1}\right)=h_{n}(1)=0$ and $g\left(\lambda_{1}\right)=g(1)=1$;

- if $j \geq 2$ and $\lambda_{j}=0$, we can set $g\left(\lambda_{j}\right)=g(0)=0$ and $h_{n}\left(\lambda_{j}\right)=h_{n}(0)$ is not relevant; 
- if $j \geq 2$ and $\lambda_{j} \neq 0$, we can set $g\left(\lambda_{j}\right)=\lambda_{j}$ and $h_{n}\left(\lambda_{j}\right)=\widehat{r}_{n-1}\left(1-\lambda_{j}\right)$ or we can set $h_{n}\left(\lambda_{j}\right)=\widehat{q}_{n, n}$ and $g\left(\lambda_{j}\right)=0 .^{1}$

Hence, since $\widehat{r}_{n-1}$ and $\widehat{q}_{n, n}$ have convergence rates $n^{\gamma}$ and $n^{\nu}$, respectively, we choose to set

$$
h_{n}(x):= \begin{cases}\widehat{r}_{n-1}(1-x) & \text { if } v<\gamma \\ \widehat{q}_{n, n} \mathbb{1}_{\{x \neq 1\}} & \text { if } v \geq \gamma\end{cases}
$$

and

$$
g(x):= \begin{cases}x & \text { if } v<\gamma \\ \mathbb{1}_{\{x=1\}} & \text { if } v \geq \gamma .\end{cases}
$$

Now, recalling that $\mathbf{v}_{j}$, for $j=1, \ldots, N$, denote the right eigenvectors of $W$, we define, for each $j=1, \ldots, N$, two vectors $\mathbf{v}_{j(1)}$ and $\mathbf{v}_{j(2)}$ of dimension $2 N$ as follows. We set $V_{j}:=\left(\mathbf{v}_{j(1)}, \mathbf{v}_{j(2)}\right)$, that is the matrix of dimension $2 N \times 2$ having $\mathbf{v}_{j(1)}$ and $\mathbf{v}_{j(2)}$ as columns, and we impose the condition

$$
V_{j}=V_{j}^{*} P_{j}^{-\top} \quad \text { where } V_{j}^{*}:=\left(\begin{array}{cc}
\mathbf{v}_{j} & \mathbf{0} \\
\mathbf{0} & \mathbf{v}_{j}
\end{array}\right) \quad \text { and } \quad P_{j}^{-\top}:=\left(\begin{array}{cc}
1 & -g\left(\lambda_{j}\right) \\
0 & 1
\end{array}\right),
$$

so that we have

$$
\mathbf{v}_{j(1)}=\left(\begin{array}{c}
\mathbf{v}_{j} \\
\mathbf{0}
\end{array}\right) \quad \text { and } \quad \mathbf{v}_{j(2)}=\left(\begin{array}{c}
-g\left(\lambda_{j}\right) \mathbf{v}_{j} \\
\mathbf{v}_{j}
\end{array}\right) .
$$

Note that, we also have

$$
V_{j}^{\top} Q_{n}=D_{Q, j, n} V_{j}^{\top}
$$

Moreover, by (2.1), we have

$$
\mathbf{u}_{j(i)}^{\top} \mathbf{v}_{j(i)}=1, \quad \text { and } \quad \mathbf{u}_{h(l)}^{\top} \mathbf{v}_{j(i)}=0, \quad \forall h \neq j \text { or } l \neq i .
$$

Finally, since $\left\{\mathbf{u}_{j(i)}: j=1, \ldots, N ; i=1,2\right\}$ and $\left\{\mathbf{v}_{j(i)}: j=1, \ldots, N ; i=1,2\right\}$ satisfy, for any $j \in\{1, \ldots, N\}$, the relation

$$
U_{j} V_{j}^{\top}=\mathbf{u}_{j(1)} \mathbf{v}_{j(1)}^{\top}+\mathbf{u}_{j(2)} \mathbf{v}_{j(2)}^{\top}=\left(\begin{array}{cc}
\mathbf{u}_{j} \mathbf{v}_{j}^{\top} & \mathbf{0} \\
\mathbf{0} & \mathbf{u}_{j} \mathbf{v}_{j}^{\top}
\end{array}\right)
$$

and since (2.3), the stochastic process $\left\{\mathbf{Y}_{n}: n \geq 1\right\}$ can be decomposed as

$$
\mathbf{Y}_{n}=\sum_{j=1}^{N} \mathbf{Y}_{j, n} \quad \text { with } \mathbf{Y}_{j, n}:=U_{j} V_{j}^{\top} \mathbf{Y}_{n}
$$

${ }^{1}$ Note that, when $v=\gamma$, by (2.5) we have $\widehat{q}_{n, n} \neq \widehat{r}_{n-1}\left(1-\lambda_{j}\right)$ for all $j \geq 2$. If there exists $j \geq 2$ such that $q=c \alpha_{j}=$ $c\left(1-\lambda_{j}\right)$, then we can set $h_{n}\left(\lambda_{j}\right)=\widehat{q}_{n, n}$ and $g\left(\lambda_{j}\right)$ is not relevant. 
The dynamics of each term $\mathbf{Y}_{j, n}$ can be deduced from (5.2) by multiplying this equation by $U_{j} V_{j}^{\top}=U_{j}^{*} V_{j}^{* \top}$ and using (5.13) and the relation $V_{j}^{\top} \mathbf{Y}_{n}=V_{j}^{\top} \mathbf{Y}_{j, n}$. We thus obtain

$$
\mathbf{Y}_{j, n}=U_{j}\left(I-D_{Q, j, n}\right) V_{j}^{\top} \mathbf{Y}_{j, n-1}+U_{j}^{*} D_{R, n} V_{j}^{* \top} \Delta \mathbf{M}_{Y, n}+U_{j} V_{j}^{\top} \Delta \mathbf{R}_{Y, n},
$$

where

$$
D_{R, n}:=\left(\begin{array}{cc}
\widehat{r}_{n-1} & 0 \\
0 & \widehat{q}_{n, n}
\end{array}\right) .
$$

For the sequel, it will be useful to decompose $\mathbf{Y}_{n}$ further as

$$
\mathbf{Y}_{n}=\sum_{j=1}^{N} \mathbf{Y}_{j, n}=\sum_{j=1}^{N} \mathbf{Y}_{j(1), n}+\sum_{j=1}^{N} \mathbf{Y}_{j(2), n}
$$

where, for any $j \in\{1, \ldots, N\}$,

$$
\begin{aligned}
\mathbf{Y}_{j, n} & =\mathbf{Y}_{j(1), n}+\mathbf{Y}_{j(2), n} \quad \text { and } \\
\mathbf{Y}_{j(i), n} & :=\mathbf{u}_{j(i)} \mathbf{v}_{j(i)}^{\top} \mathbf{Y}_{n}=\mathbf{u}_{j(i)} \mathbf{v}_{j(i)}^{\top} \mathbf{Y}_{j, n}, \quad \text { for } i=1,2
\end{aligned}
$$

and set

$$
\tilde{\mathbf{Y}}_{n}:=\mathbf{Y}_{1(1), n}=\mathbf{u}_{1(1)} \mathbf{v}_{1(1)}^{\top} \mathbf{Y}_{n}=\left(\begin{array}{c}
\mathbf{u}_{1} \mathbf{v}_{1}^{\top} \mathbf{Z}_{n} \\
\mathbf{u}_{1} \mathbf{v}_{1}^{\top} \mathbf{Z}_{n}
\end{array}\right)=\widetilde{Z}_{n}\left(\begin{array}{c}
\mathbf{1} \\
\mathbf{1}
\end{array}\right) \quad \text { with } \widetilde{Z}_{n}:=N^{-1 / 2} \mathbf{v}_{1}^{\top} Z_{n},
$$

and

$$
\begin{aligned}
\widehat{\mathbf{Y}}_{n} & :=\mathbf{Y}_{n}-\tilde{\mathbf{Y}}_{n} \\
& =\mathbf{Y}_{n}-\mathbf{Y}_{1(1), n} \\
& =\sum_{j=2}^{N} \mathbf{Y}_{j(1), n}+\sum_{j=1}^{N} \mathbf{Y}_{j(2), n} \\
& =\sum_{j=2}^{N} \mathbf{Y}_{j(1), n}+\mathbf{Y}_{1(2), n}+\sum_{j=2}^{N} \mathbf{Y}_{j(2), n} .
\end{aligned}
$$

It is worthwhile to point out that the decomposition of $\widehat{\mathbf{Y}}_{n}$ in terms of the stochastic processes $\mathbf{Y}_{j(i), n}$ is a new element with respect to the previous works and, as we will see in the sequel, it will be the key tool in order to obtain the exact convergence rate of $\widehat{\mathbf{Y}}_{n}$. Indeed, the convergence rate and the second-order asymptotic distribution of $\widehat{\mathbf{Y}}_{n}$ will be the result of the different asymptotic behaviors of the three quantities in the last term of (5.22). 


\subsection{Central limit theorems for $\tilde{\mathbf{Y}}_{n}$ and $\widehat{\mathbf{Y}}_{n}$}

The convergence rate and the second-order asymptotic distribution of $\left(\mathbf{Y}_{n}-Z_{\infty} \mathbf{1}\right)$ will be obtained by studying separately and then combining together the second-order convergence of $\widetilde{\mathbf{Y}}_{n}$ to $Z_{\infty} \mathbf{1}$ and the second-order convergence of $\widehat{\mathbf{Y}}_{n}$ to $\mathbf{0}$. To this regards, we recall that, by Aletti, Crimaldi and Ghiglietti [1], Theorem 4.2, under Assumptions 2.1 and 2.2, we have for $1 / 2<\gamma \leq 1$ that

$$
n^{\gamma-\frac{1}{2}}\left(\widetilde{\mathbf{Y}}_{n}-Z_{\infty} \mathbf{1}\right) \longrightarrow \mathcal{N}\left(\mathbf{0}, Z_{\infty}\left(1-Z_{\infty}\right) \widetilde{\Sigma}_{\gamma}\right) \text { stably in the strong sense }
$$

where $\widetilde{\Sigma}_{\gamma}$ is defined in (3.5). In this work we fully describe the second-order convergence of $\widehat{\mathbf{Y}}_{n}$, proving the following theorem.

Theorem 5.1. Under all the assumptions stated in Section 2 and recalling the definition (3.6) of $\widetilde{U}$, the following statements hold true:

(a) If $v<\gamma$, then

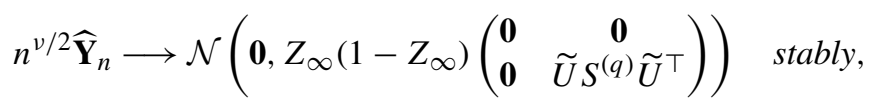

where $S^{(q)}$ is defined in (3.8).

(b) If $v=\gamma$, then

$$
n^{\gamma / 2} \widehat{\mathbf{Y}}_{n} \longrightarrow \mathcal{N}\left(\mathbf{0}, Z_{\infty}\left(1-Z_{\infty}\right)\left(\begin{array}{cc}
\widetilde{U} S_{\gamma}^{11} \widetilde{U}^{\top} & \widetilde{U} S_{\gamma}^{12} \widetilde{U}^{\top} \\
\widetilde{U} S_{\gamma}^{21} \widetilde{U}^{\top} & \widetilde{U} S_{\gamma}^{22} \widetilde{U}^{\top}
\end{array}\right)\right) \text { stably }
$$

where $S_{\gamma}^{11}, S_{\gamma}^{12}$ and $S_{\gamma}^{22}$ are known deterministic matrices, whose entries are given in the supplementary material (Aletti, Crimaldi and Ghiglietti [3], Section B), and $S_{\gamma}^{21}=\left(S_{\gamma}^{12}\right)^{\top}$.

(c) If $\gamma<v$, then

$$
n^{\gamma-\frac{v}{2}} \widehat{\mathbf{Y}}_{n} \longrightarrow \mathcal{N}\left(\mathbf{0}, Z_{\infty}\left(1-Z_{\infty}\right) \frac{c^{2}}{N\left[2 q-\mathbb{1}_{\{v=1\}}(2 \gamma-1)\right]}\left\|\mathbf{v}_{1}\right\|^{2}\left(\begin{array}{cc}
\mathbf{0} & \mathbf{0} \\
\mathbf{0} & \mathbf{1 1}^{\top}
\end{array}\right)\right) \quad \text { stably. }
$$

Remark 5.1. Note that, when $v \neq \gamma$ the convergence rates of the first and the second component of $\widehat{\mathbf{Y}}_{n}$ are always different: indeed from Aletti, Crimaldi and Ghiglietti [1], we know that, under our assumptions, the convergence rate of $\widehat{\mathbf{Z}}_{n}$ is always $n^{\gamma / 2}$, while the above theorem shows that the convergence rate of $\widehat{\mathbf{N}}_{n}$ changes according to the pair $(\gamma, v)$.

Regarding the proof of Theorem 5.1, we note that, using the definition (5.22) of $\widehat{\mathbf{Y}}_{n}$ given in Section 5.2, we can say that this random variable can be decomposed in a sum of suitable random variables that have the form

$$
\sum_{j \in J} \sum_{i \in I_{j}} \mathbf{Y}_{j(i), n}
$$


where $J \subseteq\{1, \ldots, N\}, I_{j} \subseteq\{1,2\}$ for any $j \in J$ and $Y_{j(i), n}$ is defined in (5.20). Hence, in order to characterize the asymptotic behavior of $\widehat{\mathbf{Y}}_{n}$, we first establish the second-order asymptotic behavior of the above general sum (5.24) under certain specifications of the sets $J$ and $I_{j}$ (see Lemma 5.1 below) and then we combine them together appropriately according to their convergence rates.

Lemma 5.1. Under all the assumptions stated in Section 2, consider the general sum (5.24) in the following cases:

(i) $v<\gamma, J=\{2, \ldots, N\}$ and $I_{j}=\{1\}$ for all $j \in J$;

(ii) $v<\gamma, J=\{1, \ldots, N\}$ and $I_{j}=\{2\}$ for all $j \in J$;

(iii) $v=\gamma, J=\{1, \ldots, N\}, I_{1}=\{2\}$ and $I_{j}=\{1,2\}$ for all $j \in J \backslash\{1\}$;

(iv) $\gamma<v, J=\{2, \ldots, N\}$ and $I_{j}=\{1\}$ for all $j \in J$;

(v) $\gamma<v, J=\{1\}$ and $I_{1}=\{2\}$;

(vi) $\gamma<v, J=\{2, \ldots, N\}$ and $I_{j}=\{2\}$ for all $j \in J$.

Then, in all the above listed cases, we have

$$
\begin{aligned}
& t_{n}(J(I)) \sum_{j \in J} \sum_{i \in I_{j}} \mathbf{Y}_{j(i), n} \\
& \stackrel{\text { stably }}{\longrightarrow} \mathcal{N}\left(\mathbf{0}, Z_{\infty}\left(1-Z_{\infty}\right) \sum_{j_{1} \in J} \sum_{j_{2} \in J} \mathbf{v}_{j_{1}}^{\top} \mathbf{v}_{j_{2}} \sum_{i_{1} \in I_{j_{1}}} \sum_{i_{2} \in I_{j_{2}}} d^{j_{1}\left(i_{1}\right), j_{2}\left(i_{2}\right)} \mathbf{u}_{j_{1}\left(i_{1}\right)} \mathbf{u}_{j_{2}\left(i_{2}\right)}^{\top}\right),
\end{aligned}
$$

where

$$
t_{n}(J(I)):= \begin{cases}n^{\gamma / 2} & \text { for cases (i), (iii) and (iv), } \\ n^{\nu / 2} & \text { for cases (ii) and (vi), } \\ n^{\gamma-\frac{\nu}{2}} & \text { for case (v), }\end{cases}
$$

and $d^{j_{1}\left(i_{1}\right), j_{2}\left(i_{2}\right)}$ are constants corresponding to the result of suitable limits computed in the supplementary material (Aletti, Crimaldi and Ghiglietti [3], Section A.6).

Proof of Theorem 5.1. From the above lemma, we immediately get the proof of Theorem 5.1. Indeed, in case (a) we get

$$
n^{\nu / 2} \widehat{\mathbf{Y}}_{n}=\frac{1}{n^{(\gamma-\nu) / 2}} n^{\gamma / 2} \sum_{j=2}^{N} \mathbf{Y}_{j(1), n}+n^{\nu / 2} \sum_{j=1}^{N} \mathbf{Y}_{j(2), n}
$$

where, considering the cases (i) and (ii) of Lemma 5.1, the first term in the sum converges in probability to zero, while the second term converges stably to the desired Gaussian kernel, that is the Gaussian kernel with zero mean and random covariance matrix

$$
Z_{\infty}\left(1-Z_{\infty}\right) \sum_{j_{1}=1}^{N} \sum_{j_{2}=1}^{N} \mathbf{v}_{j_{1}}^{\top} \mathbf{v}_{j_{2}} d^{j_{1}(2), j_{2}(2)} \mathbf{u}_{j_{1}(2)} \mathbf{u}_{j_{2}(2)}^{\top},
$$


where

$$
\mathbf{u}_{j_{1}(2)} \mathbf{u}_{j_{2}(2)}^{\top}=\left(\begin{array}{cc}
\mathbf{0} & \mathbf{0} \\
\mathbf{0} & \mathbf{u}_{j_{1}} \mathbf{u}_{j_{2}}^{\top}
\end{array}\right) .
$$

By means of the matrix $\widetilde{U}$, defined in (3.6), and the value of $d^{j_{1}(2), j_{2}(2)}$, computed in Aletti, Crimaldi and Ghiglietti [3], Section A.6, the above covariance matrix can be rewritten as in the statement (a) of the theorem.

In case (b), we simply have

$$
n^{\gamma / 2} \widehat{\mathbf{Y}}_{n}=n^{\gamma / 2}\left(\sum_{j=2}^{N} \mathbf{Y}_{j(1), n}+\sum_{j=1}^{N} \mathbf{Y}_{j(2), n}\right)
$$

where the right-hand term converges stably to the desired Gaussian kernel (see the case (iii) of Lemma 5.1), that is the Gaussian kernel with zero mean and random covariance matrix

$$
Z_{\infty}\left(1-Z_{\infty}\right) \sum_{j_{1}=1}^{N} \sum_{j_{2}=1}^{N} \sum_{i_{1}=1}^{2} \sum_{i_{2}=1}^{2}\left(1-\mathbb{1}_{\left\{j_{1}=i_{1}=1\right\}} \mathbb{1}_{\left\{j_{2}=i_{2}=1\right\}}\right) \mathbf{v}_{j_{1}}^{\top} \mathbf{v}_{j_{2}} d^{j_{1}\left(i_{1}\right), j_{2}\left(i_{2}\right)} \mathbf{u}_{j_{1}\left(i_{1}\right)} \mathbf{u}_{j_{2}\left(i_{2}\right)}^{\top},
$$

where

$$
\begin{aligned}
& \mathbf{u}_{j_{1}(1)} \mathbf{u}_{j_{2}(1)}^{\top}=\left(\begin{array}{cc}
\mathbf{u}_{j_{1}} \mathbf{u}_{j_{2}}^{\top} & \mathbb{1}_{\left\{j_{2}=1\right\}} \mathbf{u}_{j_{1}} \mathbf{u}_{j_{2}}^{\top} \\
\mathbb{1}_{\left\{j_{1}=1\right\}} \mathbf{u}_{j_{1}} \mathbf{u}_{j_{2}}^{\top} & \mathbb{1}_{\left\{j_{1}=1\right\}} \mathbb{1}_{\left\{j_{2}=1\right\}} \mathbf{u}_{j_{1}} \mathbf{u}_{j_{2}}^{\top}
\end{array}\right), \\
& \mathbf{u}_{j_{1}(1)} \mathbf{u}_{j_{2}(2)}^{\top}=\left(\begin{array}{cc}
\mathbf{0} & \mathbf{u}_{j_{1}} \mathbf{u}_{j_{2}}^{\top} \\
\mathbf{0} & \mathbb{1}_{\left\{j_{1}=1\right\}} \mathbf{u}_{j_{1}} \mathbf{u}_{j_{2}}^{\top}
\end{array}\right), \\
& \mathbf{u}_{j_{1}(2)} \mathbf{u}_{j_{2}(1)}^{\top}=\left(\begin{array}{cc}
\mathbf{0} & \mathbf{0} \\
\mathbf{u}_{j_{1}} \mathbf{u}_{j_{2}}^{\top} & \mathbb{1}_{\left\{j_{2}=1\right\}} \mathbf{u}_{j_{1}} \mathbf{u}_{j_{2}}^{\top}
\end{array}\right), \\
& \mathbf{u}_{j_{1}(2)} \mathbf{u}_{j_{2}(2)}^{\top}=\left(\begin{array}{cc}
\mathbf{0} & \mathbf{0} \\
\mathbf{0} & \mathbf{u}_{j_{1}} \mathbf{u}_{j_{2}}^{\top}
\end{array}\right) .
\end{aligned}
$$

Using the matrix $\widetilde{U}$, defined in (3.6), the values of $d^{j_{1}\left(i_{1}\right), j_{2}\left(i_{2}\right)}$, computed in Aletti, Crimaldi and Ghiglietti [3], Section A.6, and the entries of the matrices $S_{\gamma}^{11}, S_{\gamma}^{12}$ and $S_{\gamma}^{22}$, given in Aletti, Crimaldi and Ghiglietti [3], Section B, the above covariance matrix can be rewritten as in the statement (b) of the theorem.

Finally, in case (c), we obtain

$$
n^{\gamma-\frac{\nu}{2}} \widehat{\mathbf{Y}}_{n}=\frac{1}{n^{(\nu-\gamma) / 2}} n^{\frac{\gamma}{2}} \sum_{j=2}^{N} \mathbf{Y}_{j(1), n}+n^{\gamma-\frac{v}{2}} \mathbf{Y}_{1(2), n}+\frac{1}{n^{(\nu-\gamma)}} n^{\frac{\nu}{2}} \sum_{j=2}^{N} \mathbf{Y}_{j(2), n}
$$

where, considering the cases (iv), (v) and (vi) of Lemma 5.1, we have that the first and the third terms in the sum converge in probability to zero, while the second term converges stably to 
the desired Gaussian kernel, that is the Gaussian kernel with zero mean and random covariance matrix

$$
Z_{\infty}\left(1-Z_{\infty}\right)\left\|\mathbf{v}_{1}\right\|^{2} d^{1(2), 1(2)} \mathbf{u}_{1(2)} \mathbf{u}_{1(2)}^{\top}
$$

where

$$
\mathbf{u}_{1(2)} \mathbf{u}_{1(2)}^{\top}=\left(\begin{array}{cc}
\mathbf{0} & \mathbf{0} \\
\mathbf{0} & \mathbf{u}_{1} \mathbf{u}_{1}^{\top}
\end{array}\right)=\frac{1}{N}\left(\begin{array}{cc}
\mathbf{0} & \mathbf{0} \\
\mathbf{0} & \mathbf{1 1}^{\top}
\end{array}\right) .
$$

Using the value of $d^{1(2), 1(2)}$, computed in Aletti, Crimaldi and Ghiglietti [3], Section A.6, the above covariance matrix corresponds to the one given in the statement (c) of the theorem.

We now go on with the proof of Lemma 5.1.

Proof. Proof of Lemma 5.1 Since this proof is quite long, we split it into various steps and the technical computations and details are collected in the supplementary material Aletti, Crimaldi and Ghiglietti [3].

First step: decomposition of the general sum (5.24).

First of all, we observe that, for any set $J \subseteq\{1, \ldots, N\}$, the dynamics of $\sum_{j \in J} \mathbf{Y}_{j, n}$ can be obtained by summing up equation (5.17) for $j \in J$ :

$$
\begin{aligned}
\sum_{j \in J} \mathbf{Y}_{j, n}= & \left(\sum_{j \in J} U_{j}\left(I-D_{Q, j, n}\right) V_{j}^{\top}\right) \sum_{j \in J} \mathbf{Y}_{j, n-1}+\left(\sum_{j \in J} U_{j}^{*} D_{R, n} V_{j}^{* \top}\right) \Delta \mathbf{M}_{Y, n} \\
& +\left(\sum_{j \in J} U_{j} V_{j}^{\top}\right) \Delta \mathbf{R}_{Y, n} .
\end{aligned}
$$

Then, recalling that $\mathcal{R e}\left(\alpha_{j}\right)>0$ for each $j \geq 2$ because $\mathcal{R e}\left(\lambda_{j}\right)<1$ for each $j \geq 2$, and taking an integer $m_{0} \geq 2$ large enough such that for $n \geq m_{0}$ we have $\mathcal{R e}\left(\alpha_{j}\right) c n^{-\gamma}<1$ for each $j \geq 2$ and $q n^{-v}<1$, we can write

$$
\begin{aligned}
\sum_{j \in J} \mathbf{Y}_{j, n}= & \left(\sum_{j \in J} U_{j} A_{m_{0}, n-1}^{j} V_{j}^{\top}\right) \sum_{j \in J} \mathbf{Y}_{j, m_{0}} \\
& +\sum_{k=m_{0}}^{n-1}\left(\sum_{j \in J} U_{j} A_{k+1, n-1}^{j} V_{j}^{\top} U_{j}^{*} D_{R, k} V_{j}^{* \top}\right) \Delta \mathbf{M}_{Y, k+1} \\
& +\sum_{k=m_{0}}^{n-1}\left(\sum_{j \in J} U_{j} A_{k+1, n-1}^{j} V_{j}^{\top}\right) \Delta \mathbf{R}_{Y, k+1} \quad \text { for } n \geq m_{0},
\end{aligned}
$$


where, for any $j \in J$,

$$
A_{k+1, n-1}^{j}= \begin{cases}\prod_{m=k+1}^{n-1}\left(I-D_{Q, j, m}\right) & \text { for } m_{0} \leq k \leq n-2, \\ I & \text { for } k=n-1 .\end{cases}
$$

Setting for any $x=a_{x}+i b_{x} \in \mathbb{C}$ with $a_{x}>0$ and $1 / 2<\delta \leq 1$,

$$
\begin{aligned}
p_{k}^{\delta}(x) & :=\prod_{m=m_{0}}^{k}\left(1-\frac{x}{m^{\delta}}\right) \quad \text { for } k \geq m_{0} \text { and } \\
F_{k+1, n-1}^{\gamma}(x) & :=\frac{p_{n-1}^{\gamma}(x)}{p_{k}^{\gamma}(x)} \quad \text { for } m_{0} \leq k \leq n-1,
\end{aligned}
$$

it is easy to see that, for $j=1$, we have

$$
A_{k+1, n-1}^{1}=\left(\begin{array}{cc}
1 & 0 \\
0 & F_{k+1, n-1}^{v}(q)
\end{array}\right) \text { for } m_{0} \leq k \leq n-1
$$

and, for $j \geq 2$, after some calculations reported in the supplementary material Aletti, Crimaldi and Ghiglietti [3], Section A.2, we obtain

$$
A_{k+1, n-1}^{j}=\left(\begin{array}{cc}
F_{k+1, n-1}^{\gamma}\left(c \alpha_{j}\right) & 0 \\
\lambda_{j} G_{k+1, n-1}\left(c \alpha_{j}, q\right) & F_{k+1, n-1}^{v}(q)
\end{array}\right) \quad \text { for } m_{0} \leq k \leq n-1,
$$

where

$$
G_{k+1, n-1}(x, q):=\sum_{l=k+1}^{n-1} F_{l+1, n-1}^{\gamma}(x) h_{l}\left(1-c^{-1} x\right) F_{k+1, l-1}^{v}(q) .
$$

Then, since $V_{j}^{\top} U_{j}^{*}=P_{j}^{-1}$, equation (5.29) can be rewritten as

$$
\begin{aligned}
\sum_{j \in J} \mathbf{Y}_{j, n}= & \left(\sum_{j \in J} U_{j} A_{m_{0}, n-1}^{j} V_{j}^{\top}\right) \sum_{j \in J} \mathbf{Y}_{j, m_{0}}+\sum_{k=m_{0}}^{n-1} \mathbf{T}_{k+1, n-1}^{J} \\
& +\sum_{k=m_{0}}^{n-1} \boldsymbol{\rho}_{k+1, n-1}^{J} \quad \text { for } n \geq m_{0},
\end{aligned}
$$

with

$$
\begin{aligned}
& \mathbf{T}_{k+1, n-1}^{J}=\left(\sum_{j \in J} U_{j} A_{k+1, n-1}^{j} P_{j}^{-1} D_{R, k} V_{j}^{* \top}\right) \Delta \mathbf{M}_{Y, k+1}, \\
& \boldsymbol{\rho}_{k+1, n-1}^{J}=\left(\sum_{j \in J} U_{j} A_{k+1, n-1}^{j} V_{j}^{\top}\right) \Delta \mathbf{R}_{Y, k+1} .
\end{aligned}
$$


In order to get a similar decomposition for the general sum (5.24), we set, for any $j \in J$,

$$
\begin{aligned}
U_{j(1)}^{0} & :=\left(\begin{array}{ll}
\mathbf{u}_{j(1)} & \mathbf{0}
\end{array}\right)=\left(\begin{array}{cc}
\mathbf{u}_{j} & \mathbf{0} \\
g\left(\lambda_{j}\right) \mathbf{u}_{j} & \mathbf{0}
\end{array}\right) \text { and } \\
U_{j(2)}^{0} & :=\left(\begin{array}{ll}
\mathbf{0} & \mathbf{u}_{j(2)}
\end{array}\right)=\left(\begin{array}{cc}
\mathbf{0} & \mathbf{0} \\
\mathbf{0} & \mathbf{u}_{j}
\end{array}\right)
\end{aligned}
$$

and taking into account the last relation in (5.20), we get

$$
\begin{aligned}
\sum_{j \in J} \sum_{i \in I_{j}} \mathbf{Y}_{j(i), n}= & C_{m_{0}, n-1}^{J(I)} \sum_{j \in J} \mathbf{Y}_{j, m_{0}}+\sum_{k=m_{0}}^{n-1} \mathbf{T}_{k+1, n-1}^{J(I)} \\
& +\sum_{k=m_{0}}^{n-1} \boldsymbol{\rho}_{k+1, n-1}^{J(I)} \text { for } n \geq m_{0},
\end{aligned}
$$

with

$$
\begin{aligned}
C_{m_{0}, n-1}^{J(I)} & =\sum_{j \in J} \sum_{i \in I_{j}} U_{j(i)}^{0} A_{m_{0}, n-1}^{j} V_{j}^{\top}, \\
\mathbf{T}_{k+1, n-1}^{J(I)} & =\left(\sum_{j \in J} \sum_{i \in I_{j}} U_{j(i)}^{0} A_{k+1, n-1}^{j} P_{j}^{-1} D_{R, k} V_{j}^{* \top}\right) \Delta \mathbf{M}_{Y, k+1}, \\
\boldsymbol{\rho}_{k+1, n-1}^{J(I)} & =\left(\sum_{j \in J} \sum_{i \in I_{j}} U_{j(i)}^{0} A_{k+1, n-1}^{j} V_{j}^{\top}\right) \Delta \mathbf{R}_{Y, k+1} .
\end{aligned}
$$

In the sequel of the proof, we will establish the asymptotic behavior of the general sum (5.24) by studying separately the three terms $C_{m_{0}, n-1}^{J(I)} \sum_{j \in J} \mathbf{Y}_{j, m_{0}}, \sum_{k=m_{0}}^{n-1} \mathbf{T}_{k+1, n-1}^{J(I)}$ and $\sum_{k=m_{0}}^{n-1} \boldsymbol{\rho}_{k+1, n-1}^{J(I)}$ in the six cases (i)-(vi) specified in the statement of the considered lemma.

Second step: asymptotic behavior of $C_{m_{0}, n-1}^{J(I)} \sum_{j \in J} \mathbf{Y}_{j, m_{0}}$.

In all the six cases (i)-(vi), we have $t_{n}(J(I)) C_{m_{0}, n-1}^{J(I)} \sum_{j \in J} \mathbf{Y}_{j, m_{0}} \stackrel{\text { a.s. }}{\longrightarrow} \mathbf{0}$. The proof of this fact is given in the supplementary material (Aletti, Crimaldi and Ghiglietti [3], Section A.4).

Third step: asymptotic behavior of $\sum_{k=m_{0}}^{n-1} \rho_{k+1, n-1}^{J(I)}$.

In all the cases (i)-(v) we have $t_{n}(J(I)) \sum_{k=m_{0}}^{n-1} \boldsymbol{\rho}_{n, k}^{J(I)} \stackrel{\text { a.s. }}{\longrightarrow} \mathbf{0}$. The proof of this fact is given in the supplementary material (Aletti, Crimaldi and Ghiglietti [3], Section A.5).

Fourth step: asymptotic behavior of $\sum_{k=m_{0}}^{n-1} \mathbf{T}_{k+1, n-1}^{J(I)}$.

We aim at proving that, for each of the cases (i)-(vi), the quantity $t_{n}(J(I)) \sum_{k=m_{0}}^{n-1} \mathbf{T}_{k+1, n-1}^{J(I)}$ converges stably to the desired Gaussian kernel. For this purpose, we apply Theorem A.1. More precisely, we set $\mathcal{G}_{k, n}=\mathcal{F}_{k+1}$ and, given the fact that condition $(c 1)$ required in this theorem is obviously satisfied, we check only conditions (c2) and (c3). 
For condition (c2), we have to study the convergence of $t_{n}(J(I))^{2} \sum_{k=m_{0}}^{n-1} \mathbf{T}_{k+1, n-1}^{J(I)} \times$ $\left(\mathbf{T}_{k+1, n-1}^{J(I)}\right)^{\top}$. To this end, recalling Equation (5.38), we note that

$$
\begin{aligned}
& \sum_{k=m_{0}}^{n-1} \mathbf{T}_{k+1, n-1}^{J(I)}\left(\mathbf{T}_{k+1, n-1}^{J(I)}\right)^{\top} \\
& \quad=\sum_{j_{1} \in J, j_{2} \in J} \sum_{i_{1} \in I_{j_{1}, i_{2} \in I_{j_{2}}}} U_{j_{1}\left(i_{1}\right)}^{0}\left(\sum_{k=m_{0}}^{n-1} \mathbf{T}_{k+1, n-1}^{j_{1}}\left(\mathbf{T}_{k+1, n-1}^{j_{2}}\right)^{\top}\right) U_{j_{2}\left(i_{2}\right)}^{0, \top} \\
& \quad \sum_{j_{1} \in J, j_{2} \in J}\left[\sum_{i_{1} \in I_{j_{1}, i_{2} \in I_{j_{2}}}}^{n-1} \mathbf{T}_{k=m_{0}}^{j_{1}}\left(\mathbf{T}_{k+1, n-1}^{j_{2}}\right)^{\top}\right]_{\left(i_{1}, i_{2}\right)} \mathbf{u}_{j_{1}\left(i_{1}\right)} \mathbf{u}_{j_{2}\left(i_{2}\right)}^{\top},
\end{aligned}
$$

where $\mathbf{T}_{k+1, n-1}^{j}:=A_{k+1, n-1}^{j} P_{j}^{-1} D_{R, k} V_{j}^{* \top} \Delta \mathbf{M}_{Y, k+1}$. Thus, we can focus on the convergence of

$$
t_{n}(J(I))^{2} \sum_{k=m_{0}}^{n-1} \mathbf{T}_{k+1, n-1}^{j_{1}}\left(\mathbf{T}_{k+1, n-1}^{j_{2}}\right)^{\top} .
$$

Regarding to this, we observe that $\mathbf{T}_{k+1, n-1}^{j_{1}}\left(\mathbf{T}_{k+1, n-1}^{j_{2}}\right)^{\top}=A_{k+1, n-1}^{j_{1}} H_{k+1}^{j_{1}, j_{2}}\left(A_{k+1, n-1}^{j_{2}}\right)^{\top}$, where

$$
\begin{aligned}
H_{k+1}^{j_{1}, j_{2}} & :=P_{j_{1}}^{-1} D_{R, k} V_{j_{1}}^{* \top} \Delta \mathbf{M}_{Y, k+1} \Delta \mathbf{M}_{Y, k+1}^{\top} V_{j_{2}}^{*} D_{R, k} P_{j_{2}}^{-\top} \\
& =P_{j_{1}}^{-1} D_{R, k} V_{j_{1}}^{* \top}\left(\begin{array}{c}
I \\
I
\end{array}\right) \Delta \mathbf{M}_{k+1} \Delta \mathbf{M}_{k+1}^{\top}\left(\begin{array}{ll}
I & I
\end{array}\right) V_{j_{2}}^{*} D_{R, k} P_{j_{2}}^{-\top} \\
& =P_{j_{1}}^{-1} D_{R, k} \mathbf{1} \mathbf{v}_{j_{1}}^{\top} \Delta \mathbf{M}_{k+1} \Delta \mathbf{M}_{k+1}^{\top} \mathbf{v}_{j_{2}} \mathbf{1}^{\top} D_{R, k} P_{j_{2}}^{-\top} \\
& =\mathbf{h}_{k}^{j_{1}} \mathbf{v}_{j_{1}}^{\top} \Delta \mathbf{M}_{k+1} \Delta \mathbf{M}_{k+1}^{\top} \mathbf{v}_{j_{2}}\left(\mathbf{h}_{k}^{j_{2}}\right)^{\top} \\
& =\beta_{k+1}^{j_{1}, j_{2}} \mathbf{h}_{k}^{j_{1}}\left(\mathbf{h}_{k}^{j_{2}}\right)^{\top}
\end{aligned}
$$

with

$$
\beta_{k+1}^{j_{1}, j_{2}}:=\mathbf{v}_{j_{1}}^{\top} \Delta \mathbf{M}_{k+1} \Delta \mathbf{M}_{k+1}^{\top} \mathbf{v}_{j_{2}} \quad \text { and } \quad \mathbf{h}_{k}^{j}:=P_{j}^{-1} D_{R, k} \mathbf{1}=\left(\begin{array}{c}
\widehat{r}_{k-1} \\
\widehat{q}_{k, k}-\widehat{r}_{k-1} g\left(\lambda_{j}\right)
\end{array}\right) .
$$

Now, we set $\mathbf{d}_{k, n}^{j}:=A_{k+1, n-1}^{j} \mathbf{h}_{k}^{j}$, so that we can write

$$
\sum_{k=m_{0}}^{n-1} \mathbf{T}_{k+1, n-1}^{j_{1}}\left(\mathbf{T}_{k+1, n-1}^{j_{2}}\right)^{\top}=\sum_{k=m_{0}}^{n-1} \beta_{k+1}^{j_{1}, j_{2}} \mathbf{d}_{k, n}^{j_{1}}\left(\mathbf{d}_{k, n}^{j_{2}}\right)^{\top}
$$

Hence, in order to obtain the almost sure convergence of $t_{n}(J(I))^{2} \sum_{k=m_{0}}^{n-1} \mathbf{T}_{k+1, n-1}^{j_{1}}\left(\mathbf{T}_{k+1, n-1}^{j_{2}}\right)^{\top}$, by means of the usual martingale arguments (see Aletti, Crimaldi and Ghiglietti [2], Lemma B.1), 
it is enough to prove the convergence of $t_{n}(J(I))^{2} \sum_{k=m_{0}}^{n-1} \mathbf{d}_{k, n}^{j_{1}}\left(\mathbf{d}_{k, n}^{j_{2}}\right)^{\top}$. Indeed, since $\left\{X_{n, j}: j=\right.$ $1, \ldots, N\}$ are conditionally independent given $\mathcal{F}_{n}$, we have $E\left[\Delta M_{n, h} \Delta M_{n, j} \mid \mathcal{F}_{n-1}\right]=0$ for $h \neq j$; while, for each $j$, using the normalization $W^{\top} \mathbf{1}=\mathbf{1}$, we have

$$
E\left[\left(\Delta M_{n, j}\right)^{2} \mid \mathcal{F}_{n-1}\right]=\left(\sum_{h=1}^{N} w_{h, j} Z_{n-1, h}\right)\left(1-\sum_{h=1}^{N} w_{h, j} Z_{n-1, h}\right) \stackrel{\text { a.s. }}{\longrightarrow} Z_{\infty}\left(1-Z_{\infty}\right) .
$$

Therefore, we get $E\left[\left(\Delta \mathbf{M}_{n}\right)\left(\Delta \mathbf{M}_{n}\right)^{\top} \mid \mathcal{F}_{n-1}\right] \stackrel{\text { a.s. }}{\longrightarrow} Z_{\infty}\left(1-Z_{\infty}\right) I$ and so

$$
E\left[\beta_{n+1}^{j_{1}, j_{2}} \mid \mathcal{F}_{n}\right]=\mathbf{v}_{j_{1}}^{\top} E\left[\Delta \mathbf{M}_{n+1}\left(\Delta \mathbf{M}_{n+1}\right)^{\top} \mid \mathcal{F}_{n}\right] \mathbf{v}_{j_{2}} \stackrel{\text { a.s. }}{\longrightarrow} Z_{\infty}\left(1-Z_{\infty}\right) \mathbf{v}_{j_{1}}^{\top} \mathbf{v}_{j_{2}}
$$

from which we finally obtain

$$
\begin{aligned}
& \text { a.s.- } \lim _{n} t_{n}(J(I))^{2} \sum_{k=m_{0}}^{n-1} \mathbf{T}_{k+1, n-1}^{j_{1}}\left(\mathbf{T}_{k+1, n-1}^{j_{2}}\right)^{\top} \\
& =Z_{\infty}\left(1-Z_{\infty}\right) \mathbf{v}_{j_{1}}^{\top} \mathbf{v}_{j_{2}} \lim _{n} t_{n}(J(I))^{2} \sum_{k=m_{0}}^{n-1} \mathbf{d}_{k, n}^{j_{1}}\left(\mathbf{d}_{k, n}^{j_{2}}\right)^{\top} .
\end{aligned}
$$

In order to compute the limits in the last term of the above relation, we observe that, by means of (5.31) and (5.32), we have the following analytic expression of $\mathbf{d}_{k, n}^{j}$ :

$$
\mathbf{d}_{k, n}^{1}=A_{k+1, n-1}^{1} \mathbf{h}_{1, k}=\left(\begin{array}{c}
\widehat{r}_{k-1} \\
\left(\widehat{q}_{k, k}-\widehat{r}_{k-1}\right) F_{k+1, n-1}^{v}(q)
\end{array}\right)
$$

and, for $j \geq 2$,

$$
\begin{aligned}
\mathbf{d}_{k, n}^{j} & =A_{k+1, n-1}^{j} \mathbf{h}_{j, k} \\
& =\left(\begin{array}{c}
\widehat{r}_{k-1} F_{k+1, n-1}^{\gamma}\left(c \alpha_{j}\right) \\
\lambda_{j} \widehat{r}_{k-1} G_{k+1, n-1}\left(c \alpha_{j}, q\right)+\left(\widehat{q}_{k, k}-\widehat{r}_{k-1} g\left(\lambda_{j}\right)\right) F_{k+1, n-1}^{v}(q)
\end{array}\right) .
\end{aligned}
$$

Using these equalities, in the supplementary material (Aletti, Crimaldi and Ghiglietti [3], Section A.6), for all the considered cases (i)-(vi), we find the limit of each component of $t_{n}(J(I))^{2} \sum_{k=m_{0}}^{n-1} \mathbf{d}_{k, n}^{j_{1}}\left(\mathbf{d}_{k, n}^{j_{2}}\right)^{\top}$, that is we compute $d^{j_{1}\left(i_{1}\right), j_{2}\left(i_{2}\right)}:=\lim _{n} t_{n}(J(I))^{2} \sum_{k=m_{0}}^{n-1} d_{k, n}^{j_{1}\left(i_{1}\right)} \times$ $d_{k, n}^{j_{2}\left(i_{2}\right)}$, where $d_{k, n}^{j(1)}$ and $d_{k, n}^{j(2)}$ are, respectively, the first and the second component of $\mathbf{d}_{k, n}^{j}$ given in (5.41) and (5.42). Summing up, we have

$$
\begin{aligned}
& \sum_{k=m_{0}}^{n-1} \mathbf{T}_{k+1, n-1}^{J(I)}\left(\mathbf{T}_{k+1, n-1}^{J(I)}\right)^{\top} \\
& \stackrel{\text { a.s. }}{\longrightarrow} Z_{\infty}\left(1-Z_{\infty}\right) \sum_{j_{1} \in J, j_{2} \in J} \mathbf{v}_{j_{1}}^{\top} \mathbf{v}_{j_{2}} \sum_{i_{1} \in I_{j_{1}}, i_{2} \in I_{j_{2}}} d^{j_{1}\left(i_{1}\right), j_{2}\left(i_{2}\right)} \mathbf{u}_{j_{1}\left(i_{1}\right)} \mathbf{u}_{j_{2}\left(i_{2}\right)}^{\top} .
\end{aligned}
$$


For the check of condition (c3) of Theorem A.1, we observe that, by (5.31), (5.32), (5.33) and (5.35), taking into account the fact that in all the considered cases with $1 \in J$, that is, (ii), (iii) and (v), we have $1 \notin I_{1}$, we can write

$$
\left|\mathbf{T}_{k+1, n-1}^{J(I)}\right|=O\left(\Gamma_{k+1, n-1}^{11}\right)+O\left(\Gamma_{k+1, n-1}^{21}\right)+O\left(\Gamma_{k+1, n-1}^{22}\right),
$$

where $\Gamma_{k+1, n-1}^{11}, \Gamma_{k+1, n-1}^{21}$ and $\Gamma_{k+1, n-1}^{22}$ are the following deterministic quantities:

$$
\begin{aligned}
& \Gamma_{k+1, n-1}^{11}:=\sum_{j \in J, j \neq 1} \mathbb{1}_{\left\{1 \in I_{j}\right\}} \widehat{r}_{k-1}\left|F_{k+1, n-1}^{\gamma}\left(c \alpha_{j}\right)\right|, \\
& \Gamma_{k+1, n-1}^{21}:=\sum_{j \in J, j \neq 1} \mathbb{1}_{\left\{2 \in I_{j}\right\}} \widehat{r}_{k-1}\left|G_{k+1, n-1}\left(c \alpha_{j}, q\right)\right|, \\
& \Gamma_{k+1, n-1}^{22}:=\sum_{j \in J} \mathbb{1}_{\left\{2 \in I_{j}\right\}}\left(\widehat{r}_{k-1}+\widehat{q}_{k, k}\right)\left|F_{k+1, n-1}^{v}(q)\right| .
\end{aligned}
$$

Therefore, we find for any $u>1$

$$
\begin{aligned}
& \left(\sup _{m_{0} \leq k \leq n-1}\left|t_{n}(J(I)) \mathbf{T}_{k+1, n-1}^{J(I)}\right|\right)^{2 u} \\
& \leq t_{n}(J(I))^{2 u} \sum_{k=m_{0}}^{n-1}\left|\mathbf{T}_{k+1, n-1}^{J(I)}\right|^{2 u} \\
& \quad=t_{n}(J(I))^{2 u}\left\{\sum_{k=m_{0}}^{n-1} O\left(\left(\Gamma_{k+1, n-1}^{11}\right)^{2 u}\right)+\sum_{k=m_{0}}^{n-1} O\left(\left(\Gamma_{k+1, n-1}^{21}\right)^{2 u}\right)+\sum_{k=m_{0}}^{n-1} O\left(\left(\Gamma_{k+1, n-1}^{22}\right)^{2 u}\right)\right\} .
\end{aligned}
$$

We now analyze the last three terms. For the first one, by Lemma B.1 with $\beta=2 \gamma u, e=2 u$ and $\delta=\gamma$, we have

$$
\begin{aligned}
& \sum_{k=m_{0}}^{n-1} O\left(\left(\Gamma_{k+1, n-1}^{11}\right)^{2 u}\right) \\
& \quad=\sum_{j \in J, j \neq 1} I_{\left\{1 \in I_{j}\right\}} O\left(\sum_{k=m_{0}}^{n-1} \frac{1}{k^{2 \gamma u}}\left|F_{k+1, n-1}^{\gamma}\left(c \alpha_{j}\right)\right|^{2 u}\right) \\
& =\sum_{j \in J, j \neq 1} I_{\left\{1 \in I_{j}\right\}} \begin{cases}O\left(n^{-\gamma(2 u-1)}\right) & \text { if } 1 / 2<\gamma<1 \\
O\left(n^{-2 c a^{*} u}\right) & \text { if } \gamma=1 \text { and } 1 / 2<c a^{*}<1-(2 u)^{-1} \\
O\left(n^{-2 u+1} \ln (n)\right) & \text { if } \gamma=1 \text { and } c a^{*}=1-(2 u)^{-1} \\
O\left(n^{-2 u+1}\right) & \text { if } \gamma=1 \text { and } c a^{*}>1-(2 u)^{-1}\end{cases}
\end{aligned}
$$

For the third term, we observe that $\widehat{r}_{k-1}=O\left(\widehat{q}_{k, k}\right)$ when $v \leq \gamma$ and $\widehat{q}_{k, k}=O\left(\widehat{r}_{k-1}\right)$ when $v>\gamma$. Hence, by Lemma B.1 with $e=2 u, \delta=v$ and $\beta=2 v u$ if $v \leq \gamma$ and $\beta=2 \gamma u$ if $v>\gamma$, we get 
for the case $v \leq \gamma$

$$
\begin{aligned}
& \sum_{k=m_{0}}^{n-1} O\left(\left(\Gamma_{k+1, n-1}^{22}\right)^{2 u}\right) \\
& \quad=\sum_{j \in J} I_{\left\{2 \in I_{j}\right\}} O\left(\sum_{k=m_{0}}^{n-1} \frac{1}{k^{2 v u}}\left|F_{k+1, n-1}^{v}(q)\right|^{2 u}\right) \\
& =\sum_{j \in J} I_{\left\{2 \in I_{j}\right\}} \begin{cases}O\left(n^{-v(2 u-1)}\right) & \text { if } 1 / 2<v<1, \\
O\left(n^{-2 q u}\right) & \text { if } v=1 \text { and } 1 / 2<q<1-(2 u)^{-1} \\
O\left(n^{-2 u+1} \ln (n)\right) & \text { if } v=1 \text { and } q=1-(2 u)^{-1}, \\
O\left(n^{-2 u+1}\right) & \text { if } v=1 \text { and } q>1-(2 u)^{-1},\end{cases}
\end{aligned}
$$

and for the case $v>\gamma$

$$
\begin{aligned}
& \sum_{k=m_{0}}^{n-1} O\left(\left(\Gamma_{k+1, n-1}^{22}\right)^{2 u}\right) \\
& \quad=\sum_{j \in J} I_{\left\{2 \in I_{j}\right\}} O\left(\sum_{k=m_{0}}^{n-1} \frac{1}{k^{2 \gamma u}}\left|F_{k+1, n-1}^{v}(q)\right|^{2 u}\right) \\
& =\sum_{j \in J} I_{\left\{2 \in I_{j}\right\}} \begin{cases}O\left(n^{-2 \gamma u+v}\right) & \text { if } 1 / 2<v<1, \\
O\left(n^{-2 q u}\right) & \text { if } v=1 \text { and } 1 / 2<q<\gamma-(2 u)^{-1} \\
O\left(n^{-2 \gamma u+1} \ln (n)\right) & \text { if } v=1 \text { and } q=\gamma-(2 u)^{-1}>1 / 2 \\
O\left(n^{-2 \gamma u+1}\right) & \text { if } v=1 \text { and } q>\max \left\{1 / 2, \gamma-(2 u)^{-1}\right\}\end{cases}
\end{aligned}
$$

For the second term, we apply Lemma B.1 together with Lemma B.2 so that we get:

Case $v<\gamma$ We have $G_{k+1, n-1}\left(c \alpha_{j}, q\right)=O\left(n^{-(\gamma-v)}\left|F_{k+1, n-1}^{v}(q)\right|+k^{-(\gamma-v)}\left|F_{k+1, n-1}^{\gamma}\left(c \alpha_{j}\right)\right|\right)$ by means of Lemma B.2, and so we find

$$
\begin{aligned}
\sum_{k=m_{0}}^{n-1} O\left(\left(\Gamma_{k+1, n-1}^{21}\right)^{2 u}\right)= & \sum_{j \in J, j \neq 1} I_{\left\{2 \in I_{j}\right\}} O\left(\sum_{k=m_{0}}^{n-1} \frac{1}{k^{2 \gamma u}}\left|G_{k+1, n-1}\left(c \alpha_{j}, q\right)\right|^{2 u}\right) \\
= & \sum_{j \in J, j \neq 1} I_{\left\{2 \in I_{j}\right\}} O\left(n^{-2(\gamma-v) u} \sum_{k=m_{0}}^{n-1} \frac{1}{k^{2 \gamma u}}\left|F_{k+1, n-1}^{v}(q)\right|^{2 u}\right. \\
& \left.+\sum_{k=m_{0}}^{n-1} \frac{1}{k^{4 \gamma u-2 v u}}\left|F_{k+1, n-1}^{\gamma}\left(c \alpha_{j}\right)\right|^{2 u}\right)
\end{aligned}
$$


where, by Lemma B.1, the first term is $O\left(n^{-4 \gamma u+2 v u+v}\right)$, while for the second term we have

$$
\begin{aligned}
& \sum_{k=m_{0}}^{n-1} \frac{1}{k^{4 \gamma u-2 v u}\left|F_{k+1, n-1}^{\gamma}\left(c \alpha_{j}\right)\right|^{2 u}} \\
& \quad= \begin{cases}O\left(n^{-4 \gamma u+2 v u+\gamma}\right) & \text { if } 1 / 2<\gamma<1 \\
O\left(n^{-2 c a^{*} u}\right) & \text { if } \gamma=1 \text { and } 1 / 2<c a^{*}<2-v-(2 u)^{-1} \\
O\left(n^{-2 c a^{*} u} \ln (n)\right) & \text { if } \gamma=1 \text { and } c a^{*}=2-v-(2 u)^{-1} \\
O\left(n^{-4 u+2 v u+1}\right) & \text { if } \gamma=1 \text { and } c a^{*}>2-v-(2 u)^{-1}\end{cases}
\end{aligned}
$$

Case $v>\gamma$ We have $G_{k+1, n-1}\left(c \alpha_{j}, q\right)=O\left(n^{-(\nu-\gamma)}\left|F_{k+1, n-1}^{v}(q)\right|+k^{-(v-\gamma)}\left|F_{k+1, n-1}^{\gamma}\left(c \alpha_{j}\right)\right|\right)$ by means of Lemma B.2, and so we find

$$
\begin{aligned}
& \sum_{k=m_{0}}^{n-1} O\left(\left(\Gamma_{k+1, n-1}^{21}\right)^{2 u}\right) \\
& =\sum_{j \in J, j \neq 1} I_{\left\{2 \in I_{j}\right\}} O\left(\sum_{k=m_{0}}^{n-1} \frac{1}{k^{2 \gamma u}}\left|G_{k+1, n-1}\left(c \alpha_{j}, q\right)\right|^{2 u}\right) \\
& =\sum_{j \in J, j \neq 1} I_{\left\{2 \in I_{j}\right\}} O\left(n^{-2(\nu-\gamma) u} \sum_{k=m_{0}}^{n-1} \frac{1}{k^{2 \gamma u}}\left|F_{k+1, n-1}^{v}(q)\right|^{2 u}\right. \\
& \left.\quad+\sum_{k=m_{0}}^{n-1} \frac{1}{k^{2 v u}}\left|F_{k+1, n-1}^{\gamma}\left(c \alpha_{j}\right)\right|^{2 u}\right),
\end{aligned}
$$

where, by Lemma B.1, the second term is $O\left(n^{-2 v u+\gamma}\right)$, while the sum in the first term has the asymptotic behavior given in (5.43).

Case $v=\gamma$ By assumption (2.5) and Lemma B.2, we have ${ }^{2} G_{k+1, n-1}\left(c \alpha_{j}, q\right)=O \times$ $\left(\left|F_{k+1, n-1}^{\gamma}(q)\right|+\left|F_{k+1, n-1}^{\gamma}\left(c \alpha_{j}\right)\right|\right)$, and so we find

$$
\begin{aligned}
& \sum_{k=m_{0}}^{n-1} O\left(\left(\Gamma_{k+1, n-1}^{21}\right)^{2 u}\right) \\
& \quad=\sum_{j \in J, j \neq 1} I_{\left\{2 \in I_{j}\right\}} O\left(\sum_{k=m_{0}}^{n-1} \frac{1}{k^{2 \gamma u}}\left|F_{k+1, n-1}^{\gamma}(q)\right|^{2 u}+\sum_{k=m_{0}}^{n-1} \frac{1}{k^{2 \gamma u}}\left|F_{k+1, n-1}^{\gamma}\left(c \alpha_{j}\right)\right|^{2 u}\right),
\end{aligned}
$$

${ }^{2}$ If there exists $j \geq 2$ such that $q=c \alpha_{j}$, we have to consider the other asymptotic expression given in the supplementary material (Aletti, Crimaldi and Ghiglietti [3], Lemma A.4). 
where, by Lemma B.1, we have for $x=q$ or $x \in\left\{c \alpha_{j}: j \in J, j \neq 1\right\}$

$$
\sum_{k=m_{0}}^{n-1} \frac{1}{k^{2 \gamma u}}\left|F_{k+1, n-1}^{\gamma}(x)\right|^{2 u}= \begin{cases}O\left(n^{-\gamma(2 u-1)}\right) & \text { if } 1 / 2<v=\gamma<1, \\ O\left(n^{-2 a_{x} u}\right) & \text { if } v=\gamma=1 \text { and } 1 / 2<a_{x}<1-(2 u)^{-1} \\ O\left(n^{-2 u+1} \ln (n)\right) & \text { if } v=\gamma=1 \text { and } a_{x}=1-(2 u)^{-1} \\ O\left(n^{-2 u+1}\right) & \text { if } v=\gamma=1 \text { and } a_{x}>1-(2 u)^{-1}\end{cases}
$$

and so, setting $x^{*}:=\min \left\{q, c a^{*}\right\}$, we can write

$$
\begin{aligned}
& \sum_{k=m_{0}}^{n-1} O\left(\left(\Gamma_{k+1, n-1}^{21}\right)^{2 u}\right) \\
& \quad=\sum_{j \in J, j \neq 1} I_{\left\{2 \in I_{j}\right\}} \begin{cases}O\left(n^{-\gamma(2 u-1)}\right) & \text { if } 1 / 2<v=\gamma<1 \\
O\left(n^{-2 x^{*} u}\right) & \text { if } v=\gamma=1 \text { and } 1 / 2<x^{*}<1-(2 u)^{-1} \\
O\left(n^{-2 u+1} \ln (n)\right) & \text { if } v=\gamma=1 \text { and } x^{*}=1-(2 u)^{-1} \\
O\left(n^{-2 u+1}\right) & \text { if } v=\gamma=1 \text { and } x^{*}>1-(2 u)^{-1}\end{cases}
\end{aligned}
$$

Summing up, taking into account the conditions $c a^{*}>1 / 2$ when $\gamma=1$ and $q>1 / 2$ when $v=1$, we can conclude that in all the six cases (i)-(vi), there exists a suitable $u>1$ such that $\left(\sup _{m_{0} \leq k \leq n-1}\left|t_{n}(J(I)) \mathbf{T}_{k+1, n-1}^{J(I)}\right|\right)^{2 u}$ converges in mean to zero. This convergence trivially implies condition ( $c 3)$ of Theorem A.1.

\subsection{Proof of Theorem 3.2}

The proof of Theorem 3.2 follows by recalling that $\left(\tilde{\mathbf{Y}}_{n}-Z_{\infty} \mathbf{1}\right)=\left(\tilde{\mathbf{Y}}_{n}-Z_{\infty} \mathbf{1}\right)+\widehat{\mathbf{Y}}_{n}$, where the convergence rate for the first term is $n^{\gamma-\frac{1}{2}}$ for any parameters (see (5.23)), while the convergence rate of the second term is $n^{e}$, with $e$ specified in Theorem 5.1 according to the values of the parameters. Therefore, we can have three different cases:

- If $e<\gamma-\frac{1}{2}$, then we have

$$
n^{e}\left(\mathbf{Y}_{n}-Z_{\infty} \mathbf{1}\right)=\frac{n^{e}}{n^{\gamma-\frac{1}{2}}} n^{\gamma-\frac{1}{2}}\left(\widetilde{\mathbf{Y}}_{n}-Z_{\infty} \mathbf{1}\right)+n^{e} \widehat{\mathbf{Y}}_{n},
$$

where the first term converges in probability to zero and the second term converges stably to a certain Gaussian kernel. This occurs only in case (a) with $e=v / 2$ and $v<\gamma_{0}$.

- If $e>\gamma-\frac{1}{2}$, then we have

$$
n^{\gamma-\frac{1}{2}}\left(\mathbf{Y}_{n}-Z_{\infty} \mathbf{1}\right)=n^{\gamma-\frac{1}{2}}\left(\widetilde{\mathbf{Y}}_{n}-Z_{\infty} \mathbf{1}\right)+\frac{n^{\gamma-\frac{1}{2}}}{n^{e}} n^{e} \widehat{\mathbf{Y}}_{n},
$$

where the first term converges stably (in the strong sense) to the Gaussian kernel given in (5.23) and the second term converges in probability to zero. This occurs in case (a) with 
$e=v / 2$ and $\gamma_{0}<v<\gamma$, in case (b) with $e=\gamma / 2$ and $v=\gamma<1$ and in case (c) with $e=\gamma-v / 2$ and $\gamma<v<1$.

- If $e=\gamma-\frac{1}{2}$, then we have

$$
n^{\gamma-\frac{1}{2}}\left(\mathbf{Y}_{n}-Z_{\infty} \mathbf{1}\right)=n^{\gamma-\frac{1}{2}}\left(\widetilde{\mathbf{Y}}_{n}-Z_{\infty} \mathbf{1}\right)+n^{\gamma-\frac{1}{2}} \widehat{\mathbf{Y}}_{n}
$$

where the first term converges stably in the strong sense to the Gaussian kernel given in (5.23) and the second term is $\mathcal{F}_{n}$-measurable and it converges stably to a certain Gaussian kernel. Thus, in this case, we can apply Theorem A.2 in the Appendix. This occurs in case (a) with $e=v / 2$ and $v=\gamma_{0}<1$, in case (b) with $e=\gamma / 2$ and $v=\gamma=1$ (i.e., $v=\gamma_{0}=1$ ) and in case (c) with $e=\gamma-v / 2$ and $\gamma<v=1$ (i.e. $\gamma_{0}<v=1$ ).

Remark 5.2. As told in Remark 3.3, statements (a), (b) and (c) of Theorem 3.2 with $N=1$ (and so without the condition on $\lambda^{*}$ ) can be proven with the same proof. Specifically, it is enough to take into account that when $N=1$, we have $\widehat{Y}_{n}=Y_{1(2)}$ and $\widetilde{Z}_{n}=Z_{n}$.

\section{Proof of the results for statistical applications}

Here we prove the convergence results stated in Section 4. As we will see, the decomposition of $\mathbf{Y}_{n}$ given in Section 5.2 is a fundamental tool also for the proof of these results.

\subsection{Proof of Theorem 4.1}

For the proof of this result, we need the following lemma:

Lemma 6.1. Let us set

$$
\beta:=\frac{v}{2} \mathbb{1}_{\{\nu \leq \gamma\}}+\left(\gamma-\frac{v}{2}\right) \mathbb{1}_{\{\gamma<\nu\}} .
$$

Then, under all the assumptions stated in Section 2, we have

$$
n^{\beta} \mathbf{Y}_{1(2)} \stackrel{\text { a.s. }}{\longrightarrow} \mathcal{N}\left(\mathbf{0}, Z_{\infty}\left(1-Z_{\infty}\right) \frac{\left\|\mathbf{v}_{1}\right\|^{2}}{N} d^{1(2), 1(2)}\left(\begin{array}{cc}
\mathbf{0} & \mathbf{0} \\
\mathbf{0} & \mathbf{1 1}^{\top}
\end{array}\right)\right)
$$

where

$$
d^{1(2), 1(2)}= \begin{cases}\frac{q}{2} & \text { for } v<\gamma, \\ \frac{(q-c)^{2}}{2 q-\mathbb{1}_{\{v=1\}}} & \text { for } v=\gamma \\ \frac{c^{2}}{2 q-\mathbb{1}_{\{v=1\}}(2 \gamma-1)} & \text { for } \gamma<v .\end{cases}
$$


Proof. We observe that $\mathbf{Y}_{1(2)}$ can be written as the general sum (5.24) with $J=\{1\}$ and $I_{1}=\{2\}$. Therefore case $\gamma<v$ coincides with the case (v) of Lemma 5.1, taking into account the value $d^{1(2), 1(2)}$ computed in the supplementary material (Aletti, Crimaldi and Ghiglietti [3], Section A.6) for this case and equality (5.28). The cases $v<\gamma$ and $v=\gamma$ follows from the same arguments employed for the proof of Lemma 5.1, setting $t_{n}(J(I))=n^{v / 2}$ and using the value $d^{1(2), 1(2)}$ obtained in the supplementary material (Aletti, Crimaldi and Ghiglietti [3], Section A.6) when $v \leq \gamma$.

Remark 6.1. Note that, when $v=\gamma$ and $q=c$, we have $d^{1(2), 1(2)}=0$ and so we obtain that $n^{\beta} \mathbf{Y}_{1(2)}$ converges to $\mathbf{0}$ in probability. This means that in this case the convergence of $\mathbf{Y}_{1(2)}$ to $\mathbf{0}$ is faster than $n^{-\beta}=n^{-\gamma / 2}$.

Proof. Proof of Theorem 4.1 The convergence rate and the second-order asymptotic distribution of $\widetilde{N}_{n}$ can be obtained by combining the second-order convergences of the two stochastic processes $\widetilde{Z}_{n}$ and $\left(\widetilde{N}_{n}-\widetilde{Z}_{n}\right)$. In order to get the convergence results for these two last processes, we observe that

$$
\begin{aligned}
N^{-1 / 2} \mathbf{u}_{1}^{\top}\left(\begin{array}{ll}
\mathbf{0} & I
\end{array}\right) \widetilde{\mathbf{Y}}_{n} & =\widetilde{Z}_{n} N^{-1 / 2} \mathbf{u}_{1}^{\top} \mathbf{1}=\widetilde{Z}_{n} \quad \text { and } \\
N^{-1 / 2} \mathbf{u}_{1}^{\top}\left(\begin{array}{lll}
\mathbf{0} & I
\end{array}\right) \mathbf{Y}_{1(2), n} & =N^{-1 / 2} \mathbf{u}_{1}^{\top}\left(\begin{array}{ll}
\mathbf{0} & I
\end{array}\right) \mathbf{u}_{1(2)} \mathbf{v}_{1(2)}^{\top} \mathbf{Y}_{n} \\
& =N^{-1 / 2} \mathbf{u}_{1}^{\top}\left(\begin{array}{ll}
-\mathbf{u}_{1} \mathbf{v}_{1}^{\top} & \mathbf{u}_{1} \mathbf{v}_{1}^{\top}
\end{array}\right) \mathbf{Y}_{n} \\
& =\left(\widetilde{N}_{n}-\widetilde{Z}_{n}\right) N^{-1 / 2} \mathbf{u}_{1}^{\top} \mathbf{1}=\widetilde{N}_{n}-\widetilde{Z}_{n}
\end{aligned}
$$

(where we have used (5.21) for the first equality and relations (5.20), (5.6), (5.12), (4.1) and (2.2) for the other equalities). Hence, from the convergence result stated in (5.23) and Lemma 6.1, together with Remark 6.1 , we obtain that $\widetilde{Z}_{n}$ converges in probability to the random variable $Z_{\infty}$ with rate $n^{\gamma-1 / 2}$ and $\left(\widetilde{N}_{n}-\widetilde{Z}_{n}\right)$ converges in probability to zero with at least rate $n^{\beta}$ defined in (6.1). Then, since $\widetilde{N}_{n}=\widetilde{Z}_{n}+\left(\widetilde{N}_{n}-\widetilde{Z}_{n}\right)$, it is possible to follow analogous arguments to those used in the proof of Theorem 3.2 to combine the asymptotic behaviors of $\widetilde{Z}_{n}$ and $\left(\widetilde{N}_{n}-\widetilde{Z}_{n}\right)$. More precisely:

(a) in the case $v<\gamma_{0}$, we necessarily have $\gamma_{0}=2 \gamma-1 \leq \gamma$ (since $\gamma \leq 1$ ) and so we have $\beta=v / 2<(\gamma-1 / 2)$. Thus $\widetilde{N}_{n}$ has the same convergence rate and the same asymptotic variance as $\left(\widetilde{N}_{n}-\widetilde{Z}_{n}\right)=N^{-1 / 2} \mathbf{u}_{1}^{\top}\left(\begin{array}{ll}\mathbf{0} & I\end{array}\right) \mathbf{Y}_{1(2), n}$, that is (see Lemma 6.1) we get

$$
n^{\nu / 2}\left(\tilde{N}_{n}-Z_{\infty}\right) \longrightarrow \mathcal{N}\left(0, Z_{\infty}\left(1-Z_{\infty}\right) \widetilde{\sigma}^{2}\right) \text { stably }
$$

with $\widetilde{\sigma}^{2}=q / 2$

(b) in the case $\gamma_{0}<v<1$, we have $\beta>(\gamma-1 / 2)$ and hence $\widetilde{N}_{n}$ has the same asymptotic behavior as $\widetilde{Z}_{n}=N^{-1 / 2} \mathbf{u}_{1}^{\top}\left(\begin{array}{ll}\mathbf{0} & I\end{array}\right) \widetilde{\mathbf{Y}}_{n}$, that is (see (5.23))

$$
n^{\gamma-\frac{1}{2}}\left(\widetilde{N}_{n}-Z_{\infty}\right) \longrightarrow \mathcal{N}\left(0, Z_{\infty}\left(1-Z_{\infty}\right) \tilde{\sigma}_{\gamma}^{2}\right) \text { stably }
$$


(c) If $v=\gamma_{0}$ (i.e., $\left.v=2 \gamma-1 \leq \gamma\right)$ or $v=1$, we have $\beta=(\gamma-1 / 2)$ and hence the asymptotic behavior of $\widetilde{N}_{n}$ follows by combining the convergence results for $\left(\widetilde{N}_{n}-\widetilde{Z}_{n}\right)$ and $\widetilde{Z}_{n}$ as done in the proof of Theorem 3.2, and so we get

$$
n^{\gamma-\frac{1}{2}}\left(\tilde{N}_{n}-Z_{\infty}\right) \longrightarrow \mathcal{N}\left(0, Z_{\infty}\left(1-Z_{\infty}\right)\left(\tilde{\sigma}_{\gamma}^{2}+\tilde{\sigma}^{2}\right)\right) \text { stably }
$$

where $\widetilde{\sigma}^{2}$ is defined in (4.2).

Remark 6.2. Returning to Remark 4.1, we observe that in the proof of Theorem 4.1 the asymptotic behavior of $\widetilde{N}_{n}$ is obtained as the combination of the asymptotic behaviors of $\widetilde{N}_{n}-\widetilde{Z}_{n}$ and $\widetilde{Z}_{n}$. In case (b), $\widetilde{Z}_{n}$ converges slower than $\widetilde{N}_{n}-\widetilde{Z}_{n}$, and so only the rate and the asymptotic variance of $\widetilde{Z}_{n}$ appear in the statement of the result. However, if we look at an higher level of approximation, we should also consider the process $\widetilde{N}_{n}-\widetilde{Z}_{n}$, that converges to zero with at least rate $n^{\beta}$. Then, we can note that $\beta$ as a function of $v$ has its maximum in $v=\gamma$, which hence provides the "optimal value" of $\nu$. In addition, in this case the quantity $d^{1(2) 1(2)}$ as a function of $q$ has its minimum in $q=c$, which hence gives the "optimal value" of $q$. Note that, as told in the previous Remark 6.1, when $v=\gamma$ and $q=c$, we have $n^{\beta} \mathbf{Y}_{1(2)} \rightarrow \mathbf{0}$ in probability and so also $n^{\beta}\left(\widetilde{N}_{n}-\widetilde{Z}_{n}\right) \rightarrow 0$ in probability. This means that in this case the convergence of $\widetilde{N}_{n}-\widetilde{Z}_{n}$ to zero is faster then $n^{-\beta}=n^{-\gamma / 2}$.

\subsection{Proof of Theorem 4.2}

Recalling (4.1), together with (2.3) and the fact that

$$
U_{j}^{*} V_{j}^{* \top}=\left(\begin{array}{cc}
\mathbf{u}_{j} \mathbf{v}_{j}^{\top} & \mathbf{0} \\
\mathbf{0} & \mathbf{u}_{j} \mathbf{v}_{j}^{\top}
\end{array}\right)
$$

we can write $\mathbf{N}_{n}^{\prime}=\sum_{j=2}^{N} \mathbf{u}_{j} \mathbf{v}_{j}^{\top} \mathbf{N}_{n}=\left(\begin{array}{ll}\mathbf{0} & I\end{array}\right) \sum_{j=2}^{N} U_{j}^{*} V_{j}^{* \top} \mathbf{Y}_{n}$. Now we can use the decomposition $\mathbf{Y}_{n}=\left(\widetilde{\mathbf{Y}}_{n}+\widehat{\mathbf{Y}}_{n}\right)$ and the fact that $U_{j}^{*} V_{j}^{* \top} \widetilde{\mathbf{Y}}_{n}=\mathbf{0}$ for any $2 \leq j \leq N$ (by (2.1) and (5.21)) in order to obtain the equality

$$
\mathbf{N}_{n}^{\prime}=\left(\begin{array}{ll}
\mathbf{0} & I
\end{array}\right) \sum_{j=2}^{N} U_{j}^{*} V_{j}^{* \top} \widehat{\mathbf{Y}}_{n}
$$

Hence, the convergence rate and the second-order asymptotic distribution of $\mathbf{N}_{n}^{\prime}$ can be obtained by using the convergences stated in Theorem 5.1 or in Lemma 5.1. Specifically, case (a) follows from Theorem 5.1(a), observing that (by (2.1)) we have

$$
\left(\begin{array}{ll}
\mathbf{0} & I
\end{array}\right) \sum_{j=2}^{N} U_{j}^{*} V_{j}^{* \top}\left(\begin{array}{cc}
\mathbf{0} & \mathbf{0} \\
\mathbf{0} & \widetilde{U}
\end{array}\right)=\left(\begin{array}{ll}
\mathbf{0} & I
\end{array}\right)\left(\begin{array}{cc}
\mathbf{0} & \mathbf{0} \\
\mathbf{0} & \widetilde{U}_{-1}
\end{array}\right)=\left(\begin{array}{ll}
\mathbf{0} & \widetilde{U}_{-1}
\end{array}\right) .
$$


Case (b) follows from Theorem 5.1(b), observing that (by (2.1)) we have

$$
\left(\begin{array}{ll}
\mathbf{0} & I
\end{array}\right) \sum_{j=2}^{N} U_{j}^{*} V_{j}^{* \top}\left(\begin{array}{cc}
\widetilde{U} & \mathbf{0} \\
\mathbf{0} & \widetilde{U}
\end{array}\right)=\left(\begin{array}{ll}
\mathbf{0} & I
\end{array}\right)\left(\begin{array}{cc}
\widetilde{U}_{-1} & \mathbf{0} \\
\mathbf{0} & \widetilde{U}_{-1}
\end{array}\right)=\left(\begin{array}{ll}
\mathbf{0} & \widetilde{U}_{-1}
\end{array}\right) .
$$

Finally, case (c) cannot be obtained directly by using the convergences stated in Theorem 5.1 since in this case we have (by (2.1))

$$
\left(\begin{array}{ll}
\mathbf{0} & I
\end{array}\right) \sum_{j=2}^{N} U_{j}^{*} V_{j}^{* \top}\left(\begin{array}{l}
\mathbf{0} \\
\mathbf{1}
\end{array}\right)=N^{1 / 2}\left(\begin{array}{ll}
\mathbf{0} & I
\end{array}\right) \sum_{j=2}^{N} U_{j}^{*} V_{j}^{* \top}\left(\begin{array}{c}
\mathbf{0} \\
\mathbf{u}_{1}
\end{array}\right)=\left(\begin{array}{ll}
\mathbf{0} & I
\end{array}\right)\left(\begin{array}{l}
\mathbf{0} \\
\mathbf{0}
\end{array}\right)=\mathbf{0} .
$$

Therefore, we need to express $\mathbf{N}_{n}^{\prime}$ in the following equivalent way:

$$
\mathbf{N}_{n}^{\prime}=\left(\begin{array}{ll}
\mathbf{0} & I
\end{array}\right) \sum_{j=2}^{N} U_{j}^{*} V_{j}^{* \top} \widehat{\mathbf{Y}}_{n}=\left(\begin{array}{ll}
\mathbf{0} & I
\end{array}\right)\left(\sum_{j=2}^{N} \mathbf{Y}_{j(1), n}+\sum_{j=2}^{N} \mathbf{Y}_{j(2), n}\right),
$$

where for the last equality we have used the decomposition (5.22) of $\widehat{\mathbf{Y}}_{n}$ and the fact that $U_{j}^{*} V_{j}^{* \top} \mathbf{Y}_{1(2), n}=U_{j}^{*} V_{j}^{* \top} \mathbf{u}_{1(2)} \mathbf{v}_{1(2)}^{\top} \mathbf{Y}_{n}=\mathbf{0}$ for $2 \leq j \leq N$. Now, we recall that, in case (c), that is $v>\gamma$, we have $g\left(\lambda_{1}\right)=g(1)=1$ and $g\left(\lambda_{j}\right)=0$ for $2 \leq j \leq N$ and so we get ( $\mathbf{0} I) \mathbf{u}_{j(1)}=\mathbf{0}$ for $2 \leq j \leq N$. As a consequence, since $\mathbf{Y}_{j(1), n}=\mathbf{u}_{j(1)} \mathbf{v}_{j(1)}^{\top} \mathbf{Y}_{n}$, we have that $\left(\begin{array}{ll}\mathbf{0} & I\end{array}\right) \sum_{j=2}^{N} \mathbf{Y}_{j(1), n}=\mathbf{0}$, and the desired convergence result follows from case (vi) of Lemma 5.1.

\section{Appendix A: Stable convergence and its variants}

This brief appendix contains some basic definitions and results concerning stable convergence and its variants. For more details, we refer the reader to Crimaldi [14,16], Crimaldi, Letta and Pratelli [19], Hall and Heyde [26] and the references therein.

Let $(\Omega, \mathcal{A}, P)$ be a probability space, and let $S$ be a Polish space, endowed with its Borel $\sigma$ field. A kernel on $S$, or a random probability measure on $S$, is a collection $K=\{K(\omega): \omega \in \Omega\}$ of probability measures on the Borel $\sigma$-field of $S$ such that, for each bounded Borel real function $f$ on $S$, the map

$$
\omega \mapsto K f(\omega)=\int f(x) K(\omega)(d x)
$$

is $\mathcal{A}$-measurable. Given a sub- $\sigma$-field $\mathcal{H}$ of $\mathcal{A}$, a kernel $K$ is said $\mathcal{H}$-measurable if all the above random variables $K f$ are $\mathcal{H}$-measurable.

On $(\Omega, \mathcal{A}, P)$, let $\left(Y_{n}\right)_{n}$ be a sequence of $S$-valued random variables, let $\mathcal{H}$ be a sub- $\sigma$-field of $\mathcal{A}$, and let $K$ be a $\mathcal{H}$-measurable kernel on $S$. Then we say that $Y_{n}$ converges $\mathcal{H}$-stably to $K$, and we write $Y_{n} \longrightarrow K \mathcal{H}$-stably, if

$$
P\left(Y_{n} \in \cdot \mid H\right) \stackrel{\text { weakly }}{\longrightarrow} E[K(\cdot) \mid H] \text { for all } H \in \mathcal{H} \text { with } P(H)>0,
$$


where $K(\cdot)$ denotes the random variable defined, for each Borel set $B$ of $S$, as $\omega \mapsto K I_{B}(\omega)=$ $K(\omega)(B)$. In the case when $\mathcal{H}=\mathcal{A}$, we simply say that $Y_{n}$ converges stably to $K$ and we write $Y_{n} \longrightarrow K$ stably. Clearly, if $Y_{n} \longrightarrow K \mathcal{H}$-stably, then $Y_{n}$ converges in distribution to the probability distribution $E[K(\cdot)]$. Moreover, the $\mathcal{H}$-stable convergence of $Y_{n}$ to $K$ can be stated in terms of the following convergence of conditional expectations:

$$
E\left[f\left(Y_{n}\right) \mid \mathcal{H}\right] \stackrel{\sigma\left(L^{1}, L^{\infty}\right)}{\longrightarrow} K f
$$

for each bounded continuous real function $f$ on $S$.

In Crimaldi, Letta and Pratelli [19] the notion of $\mathcal{H}$-stable convergence is firstly generalized in a natural way replacing in (A.1) the single sub- $\sigma$-field $\mathcal{H}$ by a collection $\mathcal{G}=\left(\mathcal{G}_{n}\right)_{n}$ (called conditioning system) of sub- $\sigma$-fields of $\mathcal{A}$ and then it is strengthened by substituting the convergence in $\sigma\left(L^{1}, L^{\infty}\right)$ by the one in probability (i.e., in $L^{1}$, since $f$ is bounded). Hence, according to Crimaldi, Letta and Pratelli [19], we say that $Y_{n}$ converges to $K$ stably in the strong sense, with respect to $\mathcal{G}=\left(\mathcal{G}_{n}\right)_{n}$, if

$$
E\left[f\left(Y_{n}\right) \mid \mathcal{G}_{n}\right] \stackrel{P}{\longrightarrow} K f
$$

for each bounded continuous real function $f$ on $S$.

Finally, a strengthening of the stable convergence in the strong sense can be naturally obtained if in (A.2) we replace the convergence in probability by the almost sure convergence: given a conditioning system $\mathcal{G}=\left(\mathcal{G}_{n}\right)_{n}$, we say that $Y_{n}$ converges to $K$ in the sense of the almost sure conditional convergence, with respect to $\mathcal{G}$, if

$$
E\left[f\left(Y_{n}\right) \mid \mathcal{G}_{n}\right] \stackrel{\text { a.s. }}{\longrightarrow} K f
$$

for each bounded continuous real function $f$ on $S$. The almost sure conditional convergence has been introduced in Crimaldi [14] and, subsequently, employed by others in the urn model literature.

We now conclude this section recalling two convergence results that we need in our proofs.

From (Crimaldi and Pratelli [20], Proposition 3.1), we can get the following result.

Theorem A.1. Let $\left(\mathbf{T}_{k, n}\right)_{1 \leq k \leq k_{n}, n \geq 1}$ be a triangular array of $d$-dimensional real random vectors, such that, for each fixed $n$, the finite sequence $\left(\mathbf{T}_{k, n}\right)_{1 \leq k \leq k_{n}}$ is a martingale difference array with respect to a given filtration $\left(\mathcal{G}_{k, n}\right)_{k \geq 0}$. Moreover, let $\left(t_{n}\right)_{n}$ be a sequence of real numbers and assume that the following conditions hold:

(c1) $\mathcal{G}_{k, n} \subseteq \mathcal{G}_{k, n+1}$ for each $n$ and $1 \leq k \leq k_{n}$;

(c2) $\sum_{k=1}^{k_{n}}\left(t_{n} \mathbf{T}_{k, n}\right)\left(t_{n} \mathbf{T}_{k, n}\right)^{\top}=t_{n}^{2} \sum_{k=1}^{k_{n}} \mathbf{T}_{k, n} \mathbf{T}_{k, n}^{\top} \stackrel{P}{\longrightarrow} \Sigma$, where $\Sigma$ is a random positive semidefinite matrix;

(c3) $\sup _{1 \leq k \leq k_{n}}\left|t_{n} \mathbf{T}_{k, n}\right| \stackrel{L^{1}}{\longrightarrow} 0$.

Then $t_{n} \sum_{k=1}^{k_{n}} \mathbf{T}_{k, n}$ converges stably to the Gaussian kernel $\mathcal{N}(\mathbf{0}, \Sigma)$. 
The following result combines together a stable convergence and a stable convergence in the strong sense.

Theorem A.2 (Berti et al. [8], Lemma 1). Suppose that $C_{n}$ and $D_{n}$ are $S$-valued random variables, that $M$ and $N$ are kernels on $S$, and that $\mathcal{G}=\left(\mathcal{G}_{n}\right)_{n}$ is a filtration satisfying $\sigma\left(C_{n}\right) \subseteq \mathcal{G}_{n}$ and $\sigma\left(D_{n}\right) \subseteq \sigma\left(\bigcup_{n} \mathcal{G}_{n}\right)$ for all $n$. If $C_{n}$ stably converges to $M$ and $D_{n}$ converges to $N$ stably in the strong sense, with respect to $\mathcal{G}$, then

$$
\left(C_{n}, D_{n}\right) \longrightarrow M \otimes N \text { stably. }
$$

(Here, $M \otimes N$ is the kernel on $S \times S$ such that $(M \otimes N)(\omega)=M(\omega) \otimes N(\omega)$ for all $\omega$.

This last result contains as a special case the fact that stable convergence and convergence in probability combine well: that is, if $C_{n}$ stably converges to $M$ and $D_{n}$ converges in probability to a random variable $D$, then $\left(C_{n}, D_{n}\right)$ stably converges to $M \otimes \delta_{D}$, where $\delta_{D}$ denotes the Dirac kernel concentrated in $D$.

\section{Appendix B: Statements of some technical lemmas}

For the reader's convenience, we collect here the statements of some technical lemmas quoted in the paper. For the proofs of these results, we refer to the supplementary material Aletti, Crimaldi and Ghiglietti [3].

Given $\left(z_{n}\right)_{n},\left(z_{n}^{\prime}\right)_{n}$ two sequences of complex numbers, the notation $z_{n}=O\left(z_{n}^{\prime}\right)$ means $\left|z_{n}\right| \leq$ $C\left|z_{n}^{\prime}\right|$ for a suitable constant $C>0$ and $n$ large enough. Then, the following results hold true.

Lemma B.1 (Aletti, Crimaldi and Ghiglietti [3], Lemma A.2). Given $\beta>1$ and $e>0$, we have

$$
\sum_{k=m_{0}}^{n} \frac{1}{k^{\beta}}\left|F_{k+1, n}^{\delta}(x)\right|^{e}= \begin{cases}O\left(n^{-(\beta-\delta)}\right) & \text { if } 1 / 2<\delta<1 \\ O\left(n^{-e a_{x}}\right) & \text { if } \delta=1 \text { and } e a_{x}<\beta-1 \\ O\left(n^{-(\beta-1)} \ln (n)\right) & \text { if } \delta=1 \text { and } e a_{x}=\beta-1 \\ O\left(n^{-(\beta-1)}\right) & \text { if } \delta=1 \text { and } e a_{x}>\beta-1\end{cases}
$$

Lemma B.2 (Aletti, Crimaldi and Ghiglietti [3], Part of Lemma A.4). When $v=\gamma$, we have for $x \in \mathbb{C} \backslash\{0, q\}$

$$
G_{k+1, n-1}(x, q)=\frac{q}{x-q}\left(F_{k+1, n-1}^{\gamma}(q)-F_{k+1, n-1}^{\gamma}(x)\right) .
$$

When $v \neq \gamma$, we have for $x \in \mathbb{C} \backslash\{0\}$

$$
\begin{aligned}
G_{k+1, n-1}(x, q)= & C(x, q)\left(\frac{F_{k+1, n-1}^{v}(q)}{(n-1)^{\mu}}-\frac{F_{k+1, n-1}^{\gamma}(x)}{k^{\mu}}\right) \\
& +O\left(\frac{\left|F_{k+1, n-1}^{v}(q)\right|}{n^{2 \mu}}+\frac{\left|F_{k+1, n-1}^{\gamma}(x)\right|}{k^{2 \mu}}\right),
\end{aligned}
$$


where $\mu:=|\gamma-v|$ and

$$
C(x, q):= \begin{cases}-\frac{x}{q} & \text { if } v<\gamma \\ \frac{q}{x} & \text { if } \gamma<v .\end{cases}
$$

\section{Acknowledgements}

Giacomo Aletti is member of "Gruppo Nazionale per il Calcolo Scientifico (GNCS)" of the Italian Institute "Istituto Nazionale di Alta Matematica (INdAM)". Irene Crimaldi is member of "Gruppo Nazionale per l'Analisi Matematica, la Probabilità e le loro Applicazioni (GNAMPA)" of the Italian Institute "Istituto Nazionale di Alta Matematica (INdAM)". She is partially supported by the Italian "Programma di Attività Integrata" (PAI), project "TOol for Fighting FakEs" (TOFFE) funded by IMT School for Advanced Studies Lucca.

\section{Supplementary Material}

Supplement to "Interacting reinforced stochastic processes: Statistical inference based on the weighted empirical means" (DOI: 10.3150/19-BEJ1143SUPP; .pdf). This supplementary file provides the proofs of the technical lemmas and some computations used for the proof of Lemma 5.1 and the entries of the matrices $S_{\gamma}^{11}, S_{\gamma}^{12}$ and $S_{\gamma}^{22}$ in Theorem 5.1.

\section{References}

[1] Aletti, G., Crimaldi, I. and Ghiglietti, A. (2017). Synchronization of reinforced stochastic processes with a network-based interaction. Ann. Appl. Probab. 27 3787-3844. MR3737938 https://doi.org/10. 1214/17-AAP1296

[2] Aletti, G., Crimaldi, I. and Ghiglietti, A. (2019). Networks of reinforced stochastic processes: Asymptotics for the empirical means. Bernoulli 25 3339-3378. MR4010957 https://doi.org/10.3150/ 18-BEJ1092

[3] Aletti, G., Crimaldi, I. and Ghiglietti, A. (2020). Supplement to "Interacting Reinforced Stochastic Processes: Statistical Inference based on the Weighted Empirical Means." https://doi.org/10.3150/ 19-BEJ1143SUPP.

[4] Aletti, G. and Ghiglietti, A. (2017). Interacting generalized Friedman's urn systems. Stochastic Process. Appl. 127 2650-2678. MR3660886 https://doi.org/10.1016/j.spa.2016.12.003

[5] Aletti, G., Ghiglietti, A. and Rosenberger, W.F. (2018). Nonparametric covariate-adjusted responseadaptive design based on a functional urn model. Ann. Statist. 46 3838-3866. MR3852670 https://doi.org/10.1214/17-AOS1677

[6] Aletti, G., Ghiglietti, A. and Vidyashankar, A.N. (2018). Dynamics of an adaptive randomly reinforced urn. Bernoulli 24 2204-2255. MR3757528 https://doi.org/10.3150/17-BEJ926

[7] Benaïm, M., Benjamini, I., Chen, J. and Lima, Y. (2015). A generalized Pólya's urn with graph based interactions. Random Structures Algorithms 46 614-634. MR3346459 https://doi.org/10.1002/ rsa. 20523 
[8] Berti, P., Crimaldi, I., Pratelli, L. and Rigo, P. (2011). A central limit theorem and its applications to multicolor randomly reinforced urns. J. Appl. Probab. 48 527-546. MR2840314 https://doi.org/10. 1239/jap/1308662642

[9] Berti, P., Crimaldi, I., Pratelli, L. and Rigo, P. (2016). Asymptotics for randomly reinforced urns with random barriers. J. Appl. Probab. 53 1206-1220. MR3581252 https://doi.org/10.1017/jpr.2016.75

[10] Chen, J. and Lucas, C. (2014). A generalized Pólya's urn with graph based interactions: Convergence at linearity. Electron. Commun. Probab. 19 67. MR3269167 https://doi.org/10.1214/ECP.v19-3094

[11] Chen, M.-R. and Kuba, M. (2013). On generalized Pólya urn models. J. Appl. Probab. 50 1169-1186. MR3161380 https://doi.org/10.1239/jap/1389370106

[12] Cirillo, P., Gallegati, M. and Hüsler, J. (2012). A Pólya lattice model to study leverage dynamics and contagious financial fragility. Adv. Complex Syst. 15 1250069. MR2972682 https://doi.org/10.1142/ S0219525912500695

[13] Collevecchio, A., Cotar, C. and LiCalzi, M. (2013). On a preferential attachment and generalized Pólya's urn model. Ann. Appl. Probab. 23 1219-1253. MR3076683 https://doi.org/10.1214/ 12-AAP869

[14] Crimaldi, I. (2009). An almost sure conditional convergence result and an application to a generalized Pólya urn. Int. Math. Forum 4 1139-1156. MR2524635

[15] Crimaldi, I. (2016). Central limit theorems for a hypergeometric randomly reinforced urn. J. Appl. Probab. 53 899-913. MR3570102 https://doi.org/10.1017/jpr.2016.48

[16] Crimaldi, I. (2016). Introduzione Alla Nozione di Convergenza Stabile e sue Varianti (Introduction to the Notion of Stable Convergence and Its Variants) 57. Bologna, Italy: Unione Matematica Italiana, Monograf s.r.l. Book written in Italian.

[17] Crimaldi, I., Dai Pra, P., Louis, P.-Y. and Minelli, I.G. (2019). Synchronization and functional central limit theorems for interacting reinforced random walks. Stochastic Process. Appl. 129 70-101. MR3906991 https://doi.org/10.1016/j.spa.2018.02.012

[18] Crimaldi, I., Dai Pra, P. and Minelli, I.G. (2016). Fluctuation theorems for synchronization of interacting Pólya's urns. Stochastic Process. Appl. 126 930-947. MR3452818 https://doi.org/10.1016/j.spa. 2015.10.005

[19] Crimaldi, I., Letta, G. and Pratelli, L. (2007). A strong form of stable convergence. In Séminaire de Probabilités XL. Lecture Notes in Math. 1899 203-225. Berlin: Springer. MR2409006 https://doi.org/10.1007/978-3-540-71189-6_9

[20] Crimaldi, I. and Pratelli, L. (2005). Convergence results for multivariate martingales. Stochastic Process. Appl. 115 571-577. MR2128630 https://doi.org/10.1016/j.spa.2004.10.004

[21] Dai Pra, P., Louis, P.-Y. and Minelli, I.G. (2014). Synchronization via interacting reinforcement. J. Appl. Probab. 51 556-568. MR3217785 https://doi.org/10.1239/jap/1402578643

[22] Eggenberger, F. and Pólya, G. (1923). Über die Statistik verketteter Vorgänge. ZAMM Z. Angew. Math. Mech. 3 279-289.

[23] Fortini, S., Petrone, S. and Sporysheva, P. (2018). On a notion of partially conditionally identically distributed sequences. Stochastic Process. Appl. 128 819-846. MR3758339 https://doi.org/10.1016/j. spa.2017.06.008

[24] Ghiglietti, A. and Paganoni, A.M. (2014). Statistical properties of two-color randomly reinforced urn design targeting fixed allocations. Electron. J. Stat. 8 708-737. MR3211029 https://doi.org/10.1214/ 14-EJS899

[25] Ghiglietti, A., Vidyashankar, A.N. and Rosenberger, W.F. (2017). Central limit theorem for an adaptive randomly reinforced urn model. Ann. Appl. Probab. 27 2956-3003. MR3719951 https://doi.org/10. 1214/16-AAP1274

[26] Hall, P. and Heyde, C.C. (1980). Martingale Limit Theory and Its Application. New York-London: Academic Press [Harcourt Brace Jovanovich, Publishers]. Probability and Mathematical Statistics. MR0624435 
[27] Hayhoe, M., Alajaji, F. and Gharesifard, B. (2018). A Polya urn-based model for epidemics on networks. In 2017 American Control Conference (ACC) 358-363.

[28] Laruelle, S. and Pagès, G. (2013). Randomized urn models revisited using stochastic approximation. Ann. Appl. Probab. 23 1409-1436. MR3098437 https://doi.org/10.1214/12-aap875

[29] Lima, Y. (2016). Graph-based Pólya's urn: Completion of the linear case. Stoch. Dyn. 161660007. MR3470556 https://doi.org/10.1142/S0219493716600078

[30] Mahmoud, H.M. (2009). Pólya Urn Models. Texts in Statistical Science Series. Boca Raton, FL: CRC Press. MR2435823

[31] Mokkadem, A. and Pelletier, M. (2006). Convergence rate and averaging of nonlinear twotime-scale stochastic approximation algorithms. Ann. Appl. Probab. 16 1671-1702. MR2260078 https://doi.org/10.1214/105051606000000448

[32] Paganoni, A.M. and Secchi, P. (2004). Interacting reinforced-urn systems. Adv. in Appl. Probab. 36 791-804. MR2079914 https://doi.org/10.1239/aap/1093962234

[33] Pemantle, R. (2007). A survey of random processes with reinforcement. Probab. Surv. 4 1-79. MR2282181 https://doi.org/10.1214/07-PS094

[34] Zhang, L.-X. (2014). A Gaussian process approximation for two-color randomly reinforced urns. Electron. J. Probab. 19 86. MR3263643 https://doi.org/10.1214/EJP.v19-3432

Received February 2019 and revised June 2019 